\title{
LOCAL PROJECTIVE RESOLUTIONS AND TRANSLATION FUNCTORS FOR KAC-MOODY ALGEBRAS
}

\author{
WAYNE NEIDHARDT
}

\begin{abstract}
Let $\mathfrak{g}$ be a Kac-Moody algebra defined by a not necessarily symmetrizable generalized Cartan matrix. We define translation functors and use them to show that the multiplicities $\left(M\left(w_{1} \cdot \lambda\right): L\left(w_{2} \cdot \lambda\right)\right)$ are independent of the dominant integral weight $\lambda$, depending only on the elements of the Weyl group. In order to define the translation functors, we introduce the notion of local projective resolutions and use them to develop the machinery of homological algebra in certain categories of $\mathfrak{g}$-modules.
\end{abstract}

1. Introduction. Translation functors were introduced, in the case of a finitedimensional split semisimple Lie algebra over a field of characteristic zero, by J. C. Jantzen in [5]. These functors can be used to show that the multiplicities $\left(M\left(w_{1} \cdot \lambda\right): L\left(w_{2} \cdot \lambda\right)\right)$ of irreducible composition factors in Verma modules are independent of the dominant integral weight $\lambda$, depending only on the elements $w_{1}$ and $w_{2}$ of the Weyl group. In generalizing to the case of infinite-dimensional Kac-Moody algebras, several difficulties arise. Not every integral weight is conjugate to a dominant integral weight, so only certain translations are possible. A bigger obstacle is presented by the fact that it is possible for $(M(\lambda): L(\mu))$ to be nonzero when $\lambda$ and $\mu$ are not in the same Weyl orbit. This can occur even in the special case of a symmetrizable generalized Cartan matrix, if $\lambda$ is not conjugate to a dominant integral weight. In the symmetrizable case, however, the Casimir operator gives us enough control over this phenomenon to be able to define the translation functors without too much difficulty, in a manner analogous to that of [5]. This was done in [10], and the multiplicities $\left(M\left(w_{1} \cdot \lambda\right): L\left(w_{2} \cdot \lambda\right)\right)$ were shown to be independent of the dominant integral weight $\lambda$. If we do not assume that the generalized Cartan matrix is symmetrizable, however, then we no longer have the Casimir operator, and we must find another way to define the translation functors.

In the present paper, we define translation functors $T_{\lambda}^{\nu}$ for the case of a not necessarily symmetrizable generalized Cartan matrix, assuming that $\lambda, \nu$, and $\nu-\lambda$ are dominant integral. These functors are used to show that the multiplicities $\left(M\left(w_{1} \cdot \lambda\right): L\left(w_{2} \cdot \lambda\right)\right)$ are independent of the dominant integral weight $\lambda$.

In order to define the functors $T_{\lambda}^{\nu}$, we first define a sequence of homological functors $W_{k}^{\nu}(k=0,1, \ldots)$. The functors $W_{0}^{\nu}$ allow us to pick out the appropriate irreducible factors with highest weights in the orbit $W \cdot \nu$, and will take the place of the Casimir operator in the definition of $T_{\lambda}^{\nu}$. In order to define the functors $W_{k}^{\nu}$, we need to do some homological algebra in the appropriate category of modules, which unfortunately does not have enough projectives. Thus we are led to define

Received by the editors July 8, 1986.

1980 Mathematics Subject Classification (1985 Revision). Primary 17B10, 17B55, 17B65, 17 B70. 
the notion of local projective resolutions, and these are used to define the functors $W_{k}^{\nu}$ and to prove their homological properties.

It should be remarked that one can prove the result concerning the multiplicities $\left(M\left(w_{1} \cdot \lambda\right): L\left(w_{2} \cdot \lambda\right)\right)$ without using all of this machinery. It is to be hoped, however, that translation functors will be a useful tool in the representation theory of KacMoody algebras, as they are in the finite-dimensional case. Also, local projective resolutions may be used to define other homological functors in addition to the functors $W_{k}^{\nu}$, and the functors $W_{k}^{\nu}$ may have uses other than defining translation. In short, the tools introduced here, though not indispensable for the multipilcity result, are perhaps more interesting than the result itself.

In $\S 2$, we introduce the basic definitions and notation regarding Kac-Moody algebras. Local projective resolutions are introduced in $\S 3$, and are shown to have the usual properties one would expect of projective resolutions. $\S 4$ is devoted to the structure of modules having filtations whose factors are Verma modules. The results of $\S \S 3$ and 4 are combined to define the functors $W_{k}^{\nu}$ in $\S 5$, where they are shown to have the usual homological property of taking short exact sequences to long exact sequences. In $\S 6$, we do a deeper analysis of the functors $W_{k}^{\nu}$, and apply the results in $\S 7$ to define translation functors $T_{\lambda}^{\nu}$ and study their effect on Verma modules and irreducibles, obtaining the result on multiplicities.

2. Definitions and notation. We give here the basic definitions and notation associated with Kac-Moody algebras and their representations. We also define certain types of filtrations on modules, which will be needed in the sequel.

Let $A=\left(A_{i j}\right)$ be an $l \times l$ generalized Cartan matrix (GCM), i.e. a matrix with integer entries satisfying $A_{i i}=2$ for all $i, A_{i j} \leq 0$ for $i \neq j$, and $A_{i j}=0$ if and only if $A_{j i}=0$. Let $K$ be a field of characteristic zero, and let $\mathfrak{g}$ be any Lie algebra over $K$ satisfying

(1) $\mathfrak{g}$ is generated by a finite-dimensional abelian subalgebra $\mathfrak{h}$, called the Cartan subalgebra, and elements $e_{1}, \ldots, e_{l}, f_{1}, \ldots, f_{l}$, called simple root vectors and negative simple root vectors, respectively.

(2) There are linearly independent sets $\left\{h_{1}, \ldots, h_{l}\right\}$ in $\mathfrak{h}$ and $\left\{\alpha_{1}, \ldots, \alpha_{l}\right\}$ in $\mathfrak{h}^{*}$, the dual vector space of $\mathfrak{h}$, such that $A_{i j}=\alpha_{j}\left(h_{i}\right)$ for all $i$ and $j$. The $\alpha_{i}$ 's are called the simple roots, and the $h_{i}$ 's are called the simple coroots.

(3) $\left[e_{i}, f_{j}\right]=\delta_{i j} h_{i}$ for all $i, j=1, \ldots, l$.

(4) $\left[h, e_{i}\right]=\alpha_{i}(h) e_{i}$ and $\left[h, f_{i}\right]=-\alpha_{i}(h) f_{i}$ for all $h \in \mathfrak{h}$ and all $i=1, \ldots, l$.

(5) $\left(\operatorname{ad~} e_{i}\right)^{-A_{i j}+1}\left(e_{j}\right)=0=\left(\operatorname{ad} f_{i}\right)^{-A_{i j}+1}\left(f_{j}\right)$ for all $i \neq j$.

(6) There is an involutive antiautomorphism $\sigma: \mathfrak{g} \rightarrow \mathfrak{g}$ such that $\sigma\left(e_{i}\right)=f_{i}$ for all $i=1, \ldots, l$, and $\sigma(h)=h$ for all $h \in \mathfrak{h}$.

For each GCM $A$, such an algebra $\mathfrak{g}$ exists and is called a GCM, or Kac-Moody, Lie algebra defined by $A$. These objects were introduced in $[6,7]$.

Let $\mathfrak{n}^{+}$(respectively, $\mathfrak{n}^{-}$) denote the subalgebra of $\mathfrak{g}$ generated by $e_{1}, \ldots, e_{l}$ (respectively, $f_{1}, \ldots, f_{l}$ ). Set $\mathfrak{b}=\mathfrak{h} \oplus \mathfrak{n}^{+}$(vector space direct sum), called the Borel subalgebra of $\mathfrak{g}$.

For $i=1, \ldots, l$, let $a_{i}=K h_{i}+K e_{i}+K f_{i}$. It is easy to see from the relations that $\mathfrak{a}_{i}$ is a subalgebra of $\mathfrak{g}$ isomorphic to $\operatorname{sl}(2, K)$.

For any $\mathfrak{h}$-module $M$ and any $\lambda \in \mathfrak{h}^{*}$, let $M_{\lambda}=\{m \in M \mid h \cdot m=\lambda(h) m$ for all $h \in \mathfrak{h}\}$, called the $\lambda$-weight space of $M$; if $M_{\lambda} \neq 0$ we call $\lambda$ a weight of $M$. In case $M$ is an $\mathfrak{h}$-module satisfying $M=\bigoplus_{\lambda \in \mathfrak{h}^{*}} M_{\lambda}$, with each $M_{\lambda}$ finite dimensional, we 
call $M$ a weight module, and we write $\Pi(M)$ for the set of weights of $M$. If $M$ is a weight module, we define its character to be $\operatorname{ch} M=\sum_{\mu \in \mathfrak{h}^{*}}\left(\operatorname{dim} M_{\mu}\right) e^{\mu}$, where the $e^{\mu}$ 's are formal exponentials. Note that this discussion applies equally well to $\mathfrak{g}$-modules and $\mathfrak{b}$-modules, which may be viewed as $\mathfrak{h}$-modules by restriction.

In the sequel, whenever $M$ and $N$ are $\mathfrak{g}$-modules which are weight modules, we will write $\operatorname{Hom}(M, N)$ for the set of $\mathfrak{g}$-module homomorphisms from $M$ to $N$, and $\operatorname{Ext}(M, N)$ for the set of equivalence classes of extensions of $M$ by $N$ in the category of weight modules.

Suppose $M$ is a $\mathfrak{g}$-module. If $v \in M_{\lambda}$ is a nonzero vector satisfying $\mathfrak{n}^{+} \cdot v=0$, we call $v$ a maximal vector. If in addition $M=U(\mathfrak{g}) v$, where $U(-)$ denotes the universal enveloping algebra functor, we call $v$ a highest weight vector and call $M$ a highest weight module of weight $\lambda$. Every highest weight module is a weight module.

For any $\lambda \in \mathfrak{h}^{*}$, let $K(\lambda)$ denote the one-dimensional $\mathfrak{b}$-module whose underlying vector space is $K$ and whose $\mathfrak{b}$-module structure is given by $(h+x) \cdot k=\lambda(h) k$ for all $h \in \mathfrak{h}, x \in \mathfrak{n}^{+}$, and $k \in K$. Then the Verma module of weight $\lambda$ is the induced $\mathfrak{g}$-module $M(\lambda)=U(\mathfrak{g}) \otimes_{U(\mathfrak{b})} K(\lambda)$. It is well known that $M(\lambda)$ is the universal highest weight module of weight $\lambda$, and that $M(\lambda)$ has a unique irreducible quotient, denoted $L(\lambda)$.

Define the root lattice $Q$ to be the free abelian group with basis $\left\{\alpha_{1}, \ldots, \alpha_{l}\right\} \subseteq$ $\mathfrak{h}^{*}$, and let $Q^{+}=\left\{\sum_{i=1}^{l} k_{i} \alpha_{i} \mid k_{i} \in \mathbf{Z}_{+}\right.$for all $\left.i\right\}$, where $\mathbf{Z}_{+}$denotes the set of nonnegative integers. Define a partial order $\leq$ on $\mathfrak{h}^{*}$ by $\mu \leq \lambda$ if $\lambda-\mu \in Q^{+}$.

Let $P=\left\{\lambda \in \mathfrak{h}^{*} \mid \lambda\left(h_{i}\right) \in \mathbf{Z}\right.$ for all $\left.i\right\}$. We call $P$ the set of integral weights, and the set $P^{+}=\left\{\lambda \in \mathfrak{h}^{*} \mid \lambda\left(h_{i}\right) \in \mathbf{Z}_{+}\right.$for all $\left.i\right\}$ the set of dominant integral weights.

For each $i=1, \ldots, l$, define the linear involution $r_{i}: \mathfrak{h}^{*} \rightarrow \mathfrak{h}^{*}$ by $r_{i}(\lambda)=\lambda-$ $\lambda\left(h_{i}\right) \alpha_{i}$ for all $\lambda \in \mathfrak{h}^{*}$. The Weyl group $W$ is defined to be the subgroup of GL $\left(\mathfrak{h}^{*}\right)$ generated by $\left\{r_{1}, \ldots, r_{l}\right\}$. For any $w \in W$, we define the length of $w$, denoted $l(w)$, to be the smallest $n$ such that $w$ may be expressed as $w=r_{i_{1}} \cdots r_{i_{n}}$. Let $\rho \in \mathfrak{h}^{*}$ be a fixed element such that $\rho\left(h_{i}\right)=1$ for all $i=1, \ldots, l$. We define the dot action of $W$ on $\mathfrak{h}^{*}$ by $w \cdot \lambda=w(\lambda+\rho)-\rho$ for all $w \in W, \lambda \in \mathfrak{h}^{*}$.

We now define certain types of filtrations of modules which will be useful in the sequel.

DEFinition 2.1 Let $M$ be a weight module. By a Verma series (VS) for $M$, we mean a filtration of $M$ by submodules $0=M_{0} \subset M_{1} \subset \cdots$ such that $M=\bigcup_{i \geq 0} M_{i}$ and such that each factor $M_{i} / M_{i-1}$ is isomorphic to some Verma module $\bar{M}\left(\lambda_{i}\right)$. The filtration may be either finite or infinite. For any $\mu \in \mathfrak{h}^{*}$, if $M$ has such a VS, we define $(M: M(\mu))$ to be the number of $i$ such that $\lambda_{i}=\mu$.

DEFinition 2.2 [2]. Let $M$ be a weight module and let $\mu \in \mathfrak{h}^{*}$. By a local composition series (LCS) for $M$ at $\mu$, we mean a finite filtation of $M$ by submodules $0=M_{0} \subset M_{1} \subset \cdots \subset M_{n}=M$ such that each factor $M_{i} / M_{i-1}$ either has no weights $\geq \mu$ or is isomorphic to some $L\left(\lambda_{i}\right)$ with $\lambda_{i} \geq \mu$. If $M$ has such an LCS at $\mu$, we define $(M: L(\mu))$ to be the number of $i$ such that $\lambda_{i}=\mu$.

REMARK. In general, it is unusual for a weight module to have a VS, but, in the categories we will be using, modules always have LCS. In any case, the numbers $(M: M(\mu))$ and $(M: L(\mu))$, when they exist, do not depend on the particular filtration, as may be seen from character considerations. 
3. Projectives and local projective resolutions. We first give the basic properties of the projective modules constructed in [11]. These modules are then used to introduce the notion of a local projective resolution, and these resolutions are shown to have nice homological properties.

We will need to work in certain categories $C(\lambda)$, where $\lambda \in \mathfrak{h}^{*}$. The category $C(\lambda)$ is defined to be the full subcategory of the category of $\mathfrak{g}$-modules $M$ such that $M$ is a weight module and such that $\mu \leq \lambda$ for all $\mu \in \Pi(M)$. These categories were introduced in [11]. We note here that for any $M \in \mathrm{Ob} C(\lambda)$ and any $\mu \in \mathfrak{h}^{*}$, an LCS for $M$ at $\mu$ exists, as shown in [2, Proposition 4.2].

For the remainder of this section, we fix $\lambda \in \mathfrak{h}^{*}$. Since there is an $\mathfrak{h}$-invariant pairing of $\mathfrak{n}^{+}$and $\mathfrak{n}^{-}$, we may use the results of $[\mathbf{1 1}]$ on projectives in $C(\lambda)$. We list here those specific results which we will need.

Proposition 3.1 [11, Propositions 4.1 AND 4.5 AND CoRollary 4.2]. For each $\mu \leq \lambda$, set

$$
P(\mu)=U(\mathfrak{g}) \otimes_{U(\mathfrak{b})}\left(U\left(\mathfrak{n}^{+}\right) /\left(\bigoplus_{\mu+\alpha \nless \lambda} U\left(\mathfrak{n}^{+}\right)_{\alpha}\right) \otimes_{K} K(\mu)\right)
$$

Then, for every $M \in \mathrm{Ob} C(\lambda), \operatorname{Hom}(P(\mu), M)$ is naturally isomorphic to

$$
\operatorname{Hom}_{\mathfrak{h}}(K(\mu), M),
$$

so that $P(\mu)$ is projective in $C(\lambda)$. Furthermore, each $P(\mu)$ has a finite $V S$, with $(P(\mu): M(\nu))=\operatorname{dim} U\left(\mathfrak{n}^{+}\right)_{\nu-\mu}$ for all $\mu, \nu \leq \lambda$.

Proposition 3.2 [11, Propositions 4.5, 4.7, AND 4.8, LemMa 4.12, COROLLARY 4.13, AND THEOREM 6.2]. Let $L(\mu)$ be an irreducible object in $C(\lambda)$. Then there is a unique finitely generated indecomposable projective object in $C(\lambda)$, denoted $I(\mu)$, which maps onto $L(\mu)$. Conversely, any finitely generated indecomposable projective object in $C(\lambda)$ has a unique irreducible quotient, so that $L(\mu) \leftrightarrow I(\mu)$ gives a one-to-one correspondence between the irreducible objects in $C(\lambda)$ and the finitely generated indecomposable projective objects in $C(\lambda)$. Furthermore, $I(\mu)$ is a direct summand of $P(\mu)$, and $I(\mu)$ has a finite VS with $M(\mu)$ as the top factor, and with $(I(\mu): M(\nu))=(M(\nu): L(\mu))$ for all $\mu, \nu \leq \lambda$.

Proposition $3.3[\mathbf{1 1}$, Proposition 5.3]. For any $M \in \mathrm{Ob} C(\lambda)$ and any $\mu \leq \lambda,(M: L(\mu))=\operatorname{dim} \operatorname{Hom}(I(\mu), M)$.

Observe that $P(\mu)$ is cyclic, since it is generated by $1 \otimes \overline{1} \otimes 1$, which is of weight $\mu$. Since $I(\mu)$ is a direct summand of $P(\mu)$, it is also generated by a vector of weight $\mu$.

Although $C(\lambda)$ does not have enough projectives, there is one case in which we may construct projective resolutions, i.e. for the case of a module with a finite VS. First note that $\operatorname{dim} \operatorname{Hom}(I(\mu), M(\mu))=(M(\mu): L(\mu))=1$, so that for any nonzero $f \in \operatorname{Hom}(I(\mu), M(\mu))$, Ker $f$ is independent of the choice of $f$. We denote Ker $f$ by $S(\mu)$. Observe that if $0=M_{0} \subset M_{1} \subset \cdots \subset M_{n-1} \subset M_{n}=I(\mu)$ is a VS for $I(\mu)$, then $M_{n} / M_{n-1} \cong M(\mu)$ and $M_{n-1}=S(\mu)$. Thus, $S(\mu)$ has a finite VS, whose factors are of the form $M(\nu)$ with $\mu<\nu \leq \lambda$. 
LEMMA 3.4 [8, LEMMA 5.2]. Suppose $M \in \mathrm{Ob} C(\lambda)$ has a finite $V S 0=$ $M_{0} \subset M_{1} \subset \cdots \subset M_{n}=M$, with factors $M_{i} / M_{i-1} \cong M\left(\mu_{i}\right)$ for $i=1, \ldots, n$. Then there is an epimorphism $\phi: \bigoplus_{i=1}^{n} I\left(\mu_{i}\right) \rightarrow M$ such that $\operatorname{Ker} \phi$ has a filtration $0=S_{0} \subset S_{1} \subset \cdots \subset S_{n}=\operatorname{Ker} \phi$ with $S_{i} / S_{i-1} \cong S\left(\mu_{i}\right)$ for $i=1, \ldots, n$.

PROOF. We use induction on $n$. If $n=1$, then $M \cong M\left(\mu_{1}\right)$, and from the short exact sequence

$$
0 \rightarrow S\left(\mu_{1}\right) \rightarrow I\left(\mu_{1}\right) \rightarrow M \rightarrow 0
$$

the assertion follows.

Suppose $n>1$. By induction, there is an epimorphism $\psi: \bigoplus_{i=1}^{n-1} I\left(\mu_{i}\right) \rightarrow M_{n-1}$ and a filtration $0=S_{0} \subset S_{1} \subset \cdots \subset S_{n-1}=\operatorname{Ker} \psi$ with $S_{i} / S_{i-1} \cong S\left(\mu_{i}\right)$ for $i=1, \ldots, n-1$. Composing this with the inclusion $M_{n-1} \subset M_{n}=M$ gives a homomorphism $\bar{\psi}: \bigoplus_{i=1}^{n-1} I\left(\mu_{i}\right) \rightarrow M$, with $\operatorname{Ker} \bar{\psi}=\operatorname{Ker} \psi$ and $\operatorname{Im} \bar{\psi}=M_{n-1}$. We also have the natrual epimorphism $\pi: I\left(\mu_{n}\right) \rightarrow M\left(\mu_{n}\right) \cong M_{n} / M_{n-1}$ with $\operatorname{Ker} \pi=$ $S\left(\mu_{n}\right)$. By projectivity of $I\left(\mu_{n}\right)$, there exists $\bar{\pi}: I\left(\mu_{n}\right) \rightarrow M$ such that the following diagram commutes:

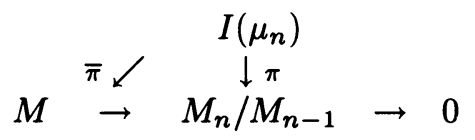

Define $\phi: \bigoplus_{i=1}^{n} I\left(\mu_{i}\right)=\left(\bigoplus_{i=1}^{n-1} I\left(\mu_{i}\right)\right) \oplus I\left(\mu_{n}\right) \rightarrow M$ by $\phi(x, y)=\bar{\psi}(x)+\bar{\pi}(y)$. This gives the following commutative diagram with exact rows:

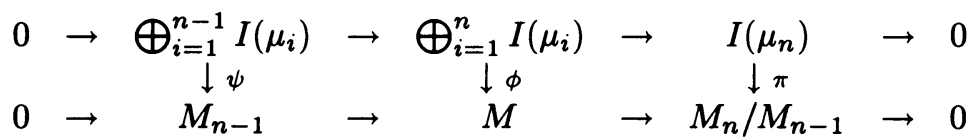

By the Short Five Lemma, $\phi$ is an epimorphism, and by the Snake Lemma we obtain an exact sequence

$$
0 \rightarrow \operatorname{Ker} \psi \rightarrow \operatorname{Ker} \phi \rightarrow \operatorname{Ker} \pi \rightarrow \operatorname{Coker} \psi=0 .
$$

But this gives a filtration $0=S_{0} \subset S_{1} \subset \cdots \subset S_{n-1}=\operatorname{Ker} \psi \subset \operatorname{Ker} \phi$ of $\operatorname{Ker} \phi$ with $S_{i} / S_{i-1} \cong S\left(\mu_{i}\right)$ for $i=1, \ldots, n-1$ and $\operatorname{Ker} \phi / \operatorname{Ker} \psi \cong \operatorname{Ker} \pi=S\left(\mu_{n}\right)$. Setting $S_{n}=\operatorname{Ker} \phi$ gives the result.

THEOREM 3.5. Let $\mu \leq \lambda$, and suppose $M \in \mathrm{Ob} C(\lambda)$ has a finite VS with factors of the form $M(\nu)$ with $\nu \geq \mu$. Then $M$ has a projective resolution in $C(\lambda)$

$$
\ldots \stackrel{d_{n+1}}{\rightarrow} P_{n} \stackrel{d_{n}}{\rightarrow} \ldots \stackrel{d_{1}}{\rightarrow} P_{0} \stackrel{d_{0}}{\rightarrow} M \rightarrow 0
$$

such that each $P_{n}$ is a finite direct sum of various $I(\nu)$ with $\nu \geq \mu$ and each $\operatorname{Ker} d_{n}=\operatorname{Im} d_{n=1}$ has a finite $V S$ of the same form as that of $M$.

PROOF. This follows easily by inducation on $n$, using the lemma and the fact that each $S(\nu)$ has such a VS.

REMARK. The resolution in the above theorem can be shown to be finite without too much effort. We will not need this fact, however.

In general, if $M \in \mathrm{Ob} C(\lambda)$, we cannot construct a projective resolution of $M$ in $C(\lambda)$, since there are not enough projectives. Thus, we introduce the notion of a local projective resolution, and prove some of its basic properties. 
DEFinition 3.6. Let $\mu \in \mathfrak{h}^{*}$ and $M \in \mathrm{Ob} C(\lambda)$. A local projective resolution of $M$ at $\mu$ is a complex

$$
\cdots \rightarrow P_{k} \rightarrow \cdots \rightarrow P_{0} \rightarrow M \rightarrow 0
$$

in $C(\lambda)$, where each $P_{k}$ is a finite direct sum of various $I(\nu)$ with $\nu \geq \mu$, and such that for any $\chi \geq \mu$, the restriction

$$
\cdots \rightarrow\left(P_{k}\right)_{\chi} \rightarrow \cdots \rightarrow\left(P_{0}\right)_{\chi} \rightarrow M_{\chi} \rightarrow 0
$$

to the $\chi$-weight spaces is exact.

Thus, a local projective resolution, although not necessarily an exact resolution, is exact "locally," i.e. above the $\mu$-weight space. The restriction we have placed on the indecomposable projectives involved is used to ensure that the projectives are generated by weight vectors above the $\mu$-weight space, a property which will allow us to do the usual homological algebra.

Proposition 3.7. For any $\mu \in \mathfrak{h}^{*}$ and $M \in \mathrm{Ob} C(\lambda)$, there exists a local projective resolution of $M$ at $\mu$.

PROOF. By [2, Proposition 4.2], there is an LCS $0=M_{0} \subset M_{1} \subset \cdots \subset M_{k}=M$ for $M$ at $\mu$. We show, by induction on $i=1, \ldots, k$, that there is a projective module $Q_{i}$ which is a finite direct sum of various $I(\nu)$ with $\nu \geq \mu$ and a map $Q_{i} \rightarrow M_{i}$ with $\left(Q_{i}\right)_{\chi} \rightarrow\left(M_{i}\right)_{\chi}$ surjective for all $\chi \geq \mu$. For $i=1$, if $M_{1}$ has no weights $\geq \mu$, we take $Q_{1}=0$. Otherwise, we have $M_{1} \cong L\left(\mu_{1}\right)$ for some $\mu_{1} \geq \mu$, and we take $Q_{1}=I\left(\mu_{1}\right)$ and $Q_{1} \rightarrow M_{1}$ to be the epimorphism given in Proposition 3.2. Now assume we have already constructed $Q_{i}$ and the map $Q_{i} \rightarrow M_{i}$ as stated, where $1 \leq i<k$. If $M_{i+1} / M_{i}$ has no weights $\geq \mu$, we take $Q_{i+1}=Q_{i}$ and $Q_{i+1} \rightarrow M_{i+1}$ to be the composition $Q_{i} \rightarrow M_{i} \subset M_{i+1}$. Otherwise, we have $M_{i+1} / M_{i} \cong L\left(\mu_{i+1}\right)$ for some $\mu_{i+1} \geq \mu$. Arguing exactly as in the proof of Lemma 3.4, we may construct the commutative diagram with exact rows

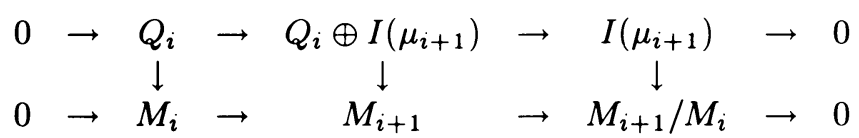

and we take $Q_{i+1}=Q_{i} \oplus I\left(\mu_{i+1}\right)$, completing the induction.

Setting $P_{0}=Q_{k}$, we have the complex $P_{0} \rightarrow M \rightarrow 0$ whose restriction to the $\chi$-weight spaces is exact for any $\chi \geq \mu$. Continuing the process with $\operatorname{Ker}\left(P_{0} \rightarrow M\right)$ in place of $M$ gives the result.

The next two propositions show that local projective resolutions may be used as basic tools for doing homological algebra in $C(\lambda)$. Their proofs are essentially the same as in the classical case of projective resolutions, with a little extra care needed since we have exactness only at certain weight spaces.

Proposition 3.8. Let $M, N \in \mathrm{Ob} C(\lambda)$, and let $\mu_{1} \geq \mu_{2}$. Suppose

$$
\cdots \rightarrow P_{k} \stackrel{d_{k}}{\rightarrow} \cdots \rightarrow P_{0} \stackrel{d_{0}}{\rightarrow} M \rightarrow 0
$$

is a local projective resolution of $M$ at $\mu_{1}$, and suppose

$$
\cdots \rightarrow Q_{k} \stackrel{d_{k}^{\prime}}{\rightarrow} \cdots \rightarrow Q_{0} \stackrel{d_{0}^{\prime}}{\rightarrow} N \rightarrow 0
$$


is a local projective resolution of $N$ at $\mu_{2}$. Given $\phi \in \operatorname{Hom}(M, N)$, there is a morphism of complexes:

$$
\begin{array}{lllllllllll}
\cdots & \rightarrow & P_{k} & \stackrel{d_{k}}{\rightarrow} & \cdots & \rightarrow & P_{0} & \stackrel{d_{0}}{\rightarrow} & M & \rightarrow & 0 \\
\phi_{k} \downarrow & & & & \phi_{0} \downarrow & & \downarrow \phi & & \\
\cdots & \rightarrow & Q_{k} & \stackrel{d_{k}^{\prime}}{\rightarrow} & \cdots & \rightarrow & Q_{0} & \stackrel{d_{0}^{\prime}}{\rightarrow} & N & \rightarrow & 0
\end{array}
$$

Furthermore, any such morphism of complexes is unique up to homotopy.

PROOF. Let $S_{0}=\sum_{\nu \geq \mu_{1}} N_{\nu}$, a vector subspace of $N$, and let $V_{0}=U(\mathfrak{g}) S_{0} \subseteq N$. Since $\mu_{1} \geq \mu_{2}$, we have $S_{0} \subseteq \operatorname{Im} d_{0}^{\prime}$ and hence $V_{0} \subseteq \operatorname{Im} d_{0}^{\prime}$. Now $P_{0}$ is the direct sum of various $I(\nu)$ with $\nu \geq \mu_{1}$, and each $I(\nu)$ is generated by a vector of weight $\nu$, which maps via $\phi \circ d_{0}$ into $S_{0}$, so that $\operatorname{Im}\left(\phi \circ d_{0}\right) \subseteq V_{0} \subseteq \operatorname{Im} d_{0}^{\prime}$. Thus, by projectivity of $P_{0}$, there exists $\phi_{0}: P_{0} \rightarrow Q_{0}$ such that $d_{0}^{\prime} \circ \phi_{0}=\phi \circ d_{0}$.

Now suppose that we have constructed $\phi_{0}, \ldots, \phi_{k-1}$ such that the diagram

$$
\begin{aligned}
& \cdots \quad \rightarrow P_{k} \stackrel{d_{k}}{\rightarrow} P_{k-1} \stackrel{d_{k-1}}{\rightarrow} \cdots \rightarrow P_{0} \stackrel{d_{0}}{\rightarrow} M \quad M \quad \rightarrow \quad 0 \\
& \cdots \rightarrow Q_{k} \stackrel{d_{k}^{\prime}}{\rightarrow} Q_{k-1} \stackrel{\phi_{k-1} \downarrow}{\stackrel{d_{k-1}^{\prime}}{\rightarrow}} \ldots \rightarrow \rightarrow Q_{0} \stackrel{d_{0}^{\prime}}{\rightarrow} N \rightarrow 0
\end{aligned}
$$

has commutative squares. Let $S_{k}=\sum_{\nu \geq \mu_{1}}\left(\operatorname{Im} d_{k}^{\prime}\right)_{\nu}=\sum_{\nu \geq \mu_{1}}\left(\operatorname{Ker} d_{k-1}^{\prime}\right)_{\nu}$, a vector subspace of $Q_{k-1}$, and let $V_{k}=U(\mathfrak{g}) S_{k} \subseteq \operatorname{Im} d_{k}^{\prime}$. Since $P_{k}$ is the direct sum of various $I(\nu)$ with $\nu \geq \mu_{1}$, and each $I(\nu)$ is generated by a vector of weight $\nu$, which maps via $\phi_{k-1} \circ d_{k}$ into $\left(\operatorname{Ker} d_{k-1}^{\prime}\right) \subseteq S_{k}$ by commutativity of the diagram, we have $\operatorname{Im}\left(\phi_{k-1} \circ d_{k}\right) \subseteq V_{k} \subseteq \operatorname{Im} d_{k}^{\prime}$. Hence, by projectivity of $P_{k}$, there exists $\phi_{k}: P_{k} \rightarrow Q_{k}$ such that $d_{k}^{\prime} \circ \phi_{k}=\phi_{k-1} \circ d_{k}$. By induction, we obtain our morphism of complexes.

It remains to show uniqueness up to homotopy. Suppose that

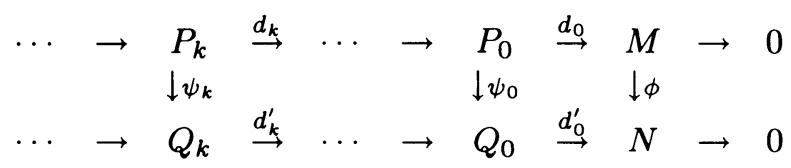

is another morphism of complexes. Then $\left(\phi_{0}-\psi_{0}\right) P_{0} \subseteq \operatorname{Ker} d_{0}^{\prime}$ by commutativity. By considering the generators of the various summands of $P_{0}$, we see that $\left(\phi_{0}-\psi_{0}\right) P_{0} \subseteq \operatorname{Im} d_{1}^{\prime}$, so that by projectivity of $P_{0}$ we obtain $h_{0}: P_{0} \rightarrow Q_{1}$ such that $d_{1}^{\prime} \circ h_{0}=\phi_{0}-\psi_{0}$. Assume we have already constructed $h_{0}, \ldots, h_{k-1}$ such that $h_{i}: P_{i} \rightarrow Q_{i+1}$ for each $i, d_{1}^{\prime} \circ h_{0}=\phi_{0}-\psi_{0}$, and $d_{i+1}^{\prime} \circ h_{i}+h_{i-1} \circ d_{i}=\phi_{i}-\psi_{i}$ for $i=1, \ldots, k-1$. Then

$$
\begin{aligned}
d_{k}^{\prime} \circ\left(\phi_{k}-\psi_{k}-h_{k-1} \circ d_{k}\right) & =d_{k}^{\prime} \circ\left(\phi_{k}-\psi_{k}\right)-d_{k}^{\prime} \circ\left(h_{k-1} \circ d_{k}\right) \\
& =\left(\phi_{k-1}-\psi_{k-1}\right) \circ d_{k}-\left(d_{k}^{\prime} \circ h_{k-1}\right) \circ d_{k} \\
& =\left(\phi_{k-1}-\psi_{k-1}-d_{k}^{\prime} \circ h_{k-1}\right) \circ d_{k} \\
& =\left(h_{k-2} \circ d_{k-1}\right) \circ d_{k}=0 .
\end{aligned}
$$

Thus, $\left(\phi_{k}-\psi_{k}-h_{k-1} \circ d_{k}\right) P_{k} \subseteq \operatorname{Ker} d_{k}^{\prime}$, and by considering generators of the summands of $P_{k}$ we have $\left(\phi_{k}-\psi_{k}-h_{k-1} \circ d_{k}\right) P_{k} \subseteq \operatorname{Im} d_{k+1}^{\prime}$. By projectivity of $P_{k}$, there exists $h_{k}: P_{k} \rightarrow Q_{k+1}$ such that $d_{k+1}^{\prime} \circ h_{k}=\phi_{k}-\psi_{k}-h_{k-1} \circ d_{k}$. That is, $d_{k+1}^{\prime} \circ h_{k}+h_{k-1} \circ d_{k}=\phi_{k}-\psi_{k}$, and by induction we obtain the homotopy. 
LEMMA 3.9. Let $\mu \in \mathfrak{h}^{*}$, and suppose

$$
0 \rightarrow M^{\prime} \stackrel{\phi}{\rightarrow} M \stackrel{\psi}{\rightarrow} M^{\prime \prime} \rightarrow 0
$$

is a sequence in $C(\lambda)$ whose restriction to the $\chi$-weight spaces is exact for every $\chi \geq \mu$. Suppose also that

$$
\cdots \stackrel{d_{1}^{\prime}}{\rightarrow} P_{0}^{\prime} \stackrel{d_{0}^{\prime}}{\rightarrow} M^{\prime} \rightarrow 0
$$

and

$$
\cdots \stackrel{d_{1}^{\prime \prime}}{\rightarrow} P_{0}^{\prime \prime} \stackrel{d_{0}^{\prime \prime}}{\rightarrow} M^{\prime \prime} \rightarrow 0
$$

are local projective resolutions of $M^{\prime}$ and $M^{\prime \prime}$, respectively, at $\mu$. Then, with $P_{0}=$ $P_{0}^{\prime} \oplus P_{0}^{\prime \prime}$, there is a commutative diagram

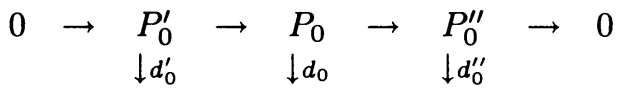

$$
\begin{aligned}
& 0 \rightarrow M^{\prime} \stackrel{\phi}{\rightarrow} M \stackrel{\psi}{\rightarrow} M^{\prime \prime} \rightarrow 0
\end{aligned}
$$

where the top row is exact (the maps being the canonical injection and projection), and the restriction of the maps in the top row gives the sequence

$$
0 \rightarrow \operatorname{Ker} d_{0}^{\prime} \rightarrow \operatorname{Ker} d_{\mathrm{c}} \rightarrow \operatorname{Ker} d_{0}^{\prime \prime} \rightarrow 0
$$

whose restriction to the $\chi$-weight spaces is exact for every $\chi \geq \mu$. Furthermore, for any $\chi \geq \mu$ we have $\left(\operatorname{Im} d_{0}\right)_{\chi}=M_{\chi}$.

Proof. Let $S=\sum_{\chi \geq \mu} M_{\chi}^{\prime \prime}$ and $V=U(\mathfrak{g}) S$. By hypothesis, $S \subseteq \operatorname{Im} \psi$ and hence $V \subseteq \operatorname{Im} \psi$. Since $P_{0}^{\prime \prime}$ is generated by vectors of various weights $\nu \geq \mu$, which map via $d_{0}^{\prime \prime}$ into $S$, we have $\operatorname{Im} d_{0}^{\prime \prime} \subseteq V \subseteq \operatorname{Im} \psi$. Thus, since $P_{0}^{\prime \prime}$ is projective, there is a map $f: P_{0}^{\prime \prime} \rightarrow M$ such that $\psi \circ f=d_{0}^{\prime \prime}$. Setting $P_{0}=P_{0}^{\prime} \oplus P_{0}^{\prime \prime}$, define $d_{0}: P_{0} \rightarrow M$ by $d_{0}(x, y)=\phi \circ d_{0}^{\prime}(x)+f(y)$. This gives the commutative diagram (3.1), with top row exact. Also, for any $\chi \geq \mu$, restricting (3.1) to the $\chi$-weight spaces and applying the Snake Lemma gives the exact sequence

$$
\begin{aligned}
& 0 \rightarrow\left(\operatorname{Ker} d_{0}^{\prime}\right)_{\chi} \rightarrow\left(\operatorname{Ker} d_{0}\right)_{\chi} \rightarrow\left(\operatorname{Ker} d_{0}^{\prime \prime}\right)_{\chi} \\
& \rightarrow\left(\text { Coker } d_{0}^{\prime}\right)_{\chi} \rightarrow\left(\text { Coker } d_{0}\right)_{\chi} \rightarrow\left(\text { Coker } d_{0}^{\prime \prime}\right)_{\chi} \rightarrow 0 \text {. }
\end{aligned}
$$

By hypothesis, $\left(\text { Coker } d_{0}^{\prime}\right)_{\chi}=0=\left(\text { Coker } d_{0}^{\prime \prime}\right)_{\chi}$. Using this in (3.2) gives two consequences: $\left(\text { Coker } d_{0}\right)_{\chi}=0$ and there is a short exact sequence

$$
0 \rightarrow\left(\operatorname{Ker} d_{0}^{\prime}\right)_{\chi} \rightarrow\left(\operatorname{Ker} d_{0}\right)_{\chi} \rightarrow\left(\operatorname{Ker} d_{0}^{\prime \prime}\right)_{\chi} \rightarrow 0,
$$

which are the desired conclusions.

Proposition 3.10. Let $\mu \in \mathfrak{h}^{*}$, and suppose

$$
0 \rightarrow M^{\prime} \stackrel{\phi}{\rightarrow} M \stackrel{\psi}{\rightarrow} M^{\prime \prime} \rightarrow 0
$$

is a sequence in $C(\lambda)$ whose restriction to the $\chi$-weight spaces is exact for every $\chi \geq \mu$. Suppose also that

$$
\cdots \rightarrow P_{n}^{\prime} \stackrel{d_{n}^{\prime}}{\rightarrow} \cdots \rightarrow P_{0}^{\prime} \stackrel{d_{0}^{\prime}}{\rightarrow} M^{\prime} \rightarrow 0
$$

and

$$
\cdots \rightarrow P_{n}^{\prime \prime} \stackrel{d_{n}^{\prime \prime}}{\rightarrow} \cdots \rightarrow P_{0}^{\prime \prime} \stackrel{d_{0}^{\prime \prime}}{\rightarrow} M^{\prime \prime} \rightarrow 0
$$


are local projective resolutions of $M^{\prime}$ and $M^{\prime \prime}$, respectively, at $\mu$. Then, setting $P_{n}=P_{n}^{\prime} \oplus P_{n}^{\prime \prime}$ for each $n$, there exist maps $d_{0}: P_{0} \rightarrow M$ and $d_{n}: P_{n} \rightarrow P_{n-1}$ $(n=1,2, \ldots)$ such that

$$
\cdots \rightarrow P_{n} \stackrel{d_{n}}{\rightarrow} \cdots \rightarrow P_{0} \stackrel{d_{0}}{\rightarrow} M \rightarrow 0
$$

is a local projective resolution of $M$ at $\mu$, and

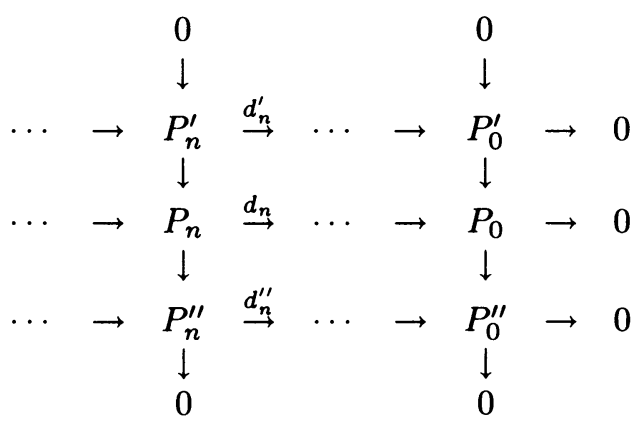

is a short exact sequence of complexes, where the vertical maps are the canonical injections and projections.

Proof. We define $d_{0}: P_{0} \rightarrow M$ as in the lemma. Note that

$$
\cdots \rightarrow P_{n}^{\prime} \stackrel{d_{n}^{\prime}}{\rightarrow} \cdots \rightarrow P_{1}^{\prime} \stackrel{d_{1}^{\prime}}{\rightarrow} \operatorname{Ker} d_{0}^{\prime} \rightarrow 0
$$

and

$$
\cdots \rightarrow P_{n}^{\prime \prime} \stackrel{d_{n}^{\prime \prime}}{\rightarrow} \cdots \rightarrow P_{1}^{\prime \prime} \stackrel{d_{1}^{\prime \prime}}{\rightarrow} \operatorname{Ker} d_{0}^{\prime \prime} \rightarrow 0
$$

are local projective resolutions of $\operatorname{Ker} d_{0}^{\prime}$ and $\operatorname{Ker} d_{0}^{\prime \prime}$, respectively, at $\mu$, and that

$$
0 \rightarrow \operatorname{Ker} d_{0}^{\prime} \rightarrow \operatorname{Ker} d_{0} \rightarrow \operatorname{Ker} d_{0}^{\prime \prime} \rightarrow 0
$$

satisfies the same hypotheses as the original sequence, by the lemma. Thus, we may define $d_{1}, d_{2}, \ldots$ inductively.

4. Modules with Verma series. In this section we prove certain results regarding the structure of modules having a VS. Note that since the projective modules occurring in local projective resolutions have VS, these results may be applied to the projectives. This will be done in the next section to define the homological functors.

We begin by stating some known properties of Verma modules.

Proposition 4.1 [9, Propositions 2.1 AND 2.4 AND LEMMA 3.1]. Let $\mu, \nu \in \mathfrak{h}^{*}$. Then $(M(\nu): L(\mu)) \neq 0$ if and only if $\operatorname{Hom}(M(\mu), M(\nu)) \neq 0$ (i.e. there is an imbedding of $M(\mu)$ in $M(\nu))$. Also, if $M$ is a highest weight module of weight $\nu$ and $\operatorname{Ext}(M(\mu), M) \neq 0$, then $(M(\nu): L(\mu)) \neq 0$ and $\nu>\mu$.

LEMMA 4.2. Let $0=M_{0} \subset M_{1} \subset \cdots \subset M_{n}=M$ be a finite $V S$ for the weight module $M$, with factors $F_{i}=M_{i} / M_{i-1} \cong M\left(\lambda_{i}\right)$ for $i=1, \ldots, n$. If 
$\operatorname{Ext}\left(M\left(\lambda_{n}\right), M\left(\lambda_{i}\right)\right)=0$ for all $i<n$, then there is another $V S 0=M_{0}^{\prime} \subset M_{1}^{\prime} \subset$ $\cdots \subset M_{n}^{\prime}=M$ for $M$ with the same length, such that $M_{1}^{\prime} \cong M\left(\lambda_{n}\right)$ and $M_{i+1}^{\prime} / M_{i}^{\prime} \cong$ $M_{i} / M_{i-1}=F_{i}$ for $i=1, \ldots, n-1$.

ProOF. Since $\operatorname{Ext}\left(M\left(\lambda_{n}\right), F_{i}\right)=0$ for all $i<n$, we have $\operatorname{Ext}\left(M\left(\lambda_{n}\right), M_{n-1}\right)=$ 0 . Thus, the short exact sequence

$$
0 \rightarrow M_{n-1} \rightarrow M_{n} \rightarrow M\left(\lambda_{n}\right) \rightarrow 0
$$

must split. Let $\phi: M\left(\lambda_{n}\right) \rightarrow M_{n}$ be a splitting map. Then, setting $M_{1}^{\prime}=\operatorname{Im} \phi$ and setting $M_{i+1}^{\prime}=M_{i} \oplus M_{1}^{\prime}$ for $i=1, \ldots, n-1$ gives the result.

PROPOSITION 4.3. Let $M$ be a weight module with $V S 0=M_{0} \subset M_{1} \subset \cdots$ (possibly infinite), with factors $F_{i}=M_{i} / M_{i-1} \cong M\left(\lambda_{i}\right)$, and let $S$ be any subset of $\mathfrak{h}^{*}$ such that $\lambda_{i} \in S$ for all $i \geq 1$. Suppose $S=S_{1} \cup S_{2}$ is a disjoint union and that $\operatorname{Hom}(M(\mu), M(\nu))=0$ whenever $\mu \in S_{1}$ and $\nu \in S_{2}$. Then $M$ has a submodule $N$ such that $N$ has a VS whose factors are precisely those $F_{i}$ with $\lambda_{i} \in S_{1}$ and $M / N$ has a VS whose factors are precisely those $F_{i}$ with $\lambda_{i} \in S_{2}$.

ProOF. Observe that, by Proposition 4.1, we also have $\operatorname{Ext}(M(\mu), M(\nu))=0$ whenever $\mu \in S_{1}$ and $\nu \in S_{2}$. We first show, by induction on $n$, that each $M_{n}$ has a submodule $N_{n}$ such that the conclusion holds for $N_{n}$ and $M_{n} / N_{n}$ (taking only those $F_{i}$ with $\left.i \leq n\right)$. For $n=1$, if $\lambda_{1} \in S_{1}$ then take $N_{1}=M_{1}$ and otherwise take $N_{1}=0$. Suppose $n>1$ and that we have $N_{n-1} \subset M_{n-1}$ as stated. By taking preimages of the VS for $M_{n-1} / N_{n-1}$, we have $N_{n-1}=V_{0} \subset V_{1} \subset \cdots \subset V_{k}=M_{n-1} \subset M_{n}$ with each $V_{i} / V_{i-1} \cong M\left(\mu_{i}\right)$ for some $\mu_{i} \in S_{2}$, and $M_{n} / M_{n-1}=M\left(\lambda_{n}\right)$. Thus, if $\lambda_{n} \in S_{2}$ we are done by taking $N_{n}=N_{n-1}$. Otherwise, if $\lambda_{n} \in S_{1}$, then applying the lemma to the VS $0=V_{0} / N_{n-1} \subset V_{1} / N_{n-1} \subset \cdots \subset V_{k} / N_{n-1} \subset M_{n} / N_{n-1}$ for $M_{n} / N_{n-1}$ and taking preimages gives $N_{n-1}=V_{0}^{\prime} \subset V_{1}^{\prime} \subset \cdots \subset V_{k}^{\prime} \subset V_{k+1}^{\prime}=M_{n}$ such that $V_{1}^{\prime} / N_{n-1} \cong M\left(\lambda_{n}\right)$ and each $V_{i+1}^{\prime} / V_{i}^{\prime} \cong V_{i} / V_{i-1}$ for $i=1, \ldots, k$, and we are done by taking $N_{n}=V_{1}^{\prime}$. This completes the induction on $n$.

In the case where the VS is infinite, observe that $N_{n-1} \subseteq N_{n}$ for each $n=1,2, \ldots$. (either equality holds when $\lambda_{n} \in S_{2}$, or else one Verma factor is added at the top when $\lambda_{n} \in S_{1}$ ) and that there are monomorphisms $M_{n-1} / N_{n-1} \rightarrow M_{n} / N_{n}$ for each $n=1,2, \ldots$ (either they are isomorphic when $\lambda_{n} \in S_{1}$, or else one Verma factor is added at the top when $\lambda_{n} \in S_{2}$ ). Thus, taking $N=\bigcup_{n \geq 0} N_{n}$ gives the result. ( $N$ has the appropriate VS by definition, and $M / N$ has the appropriate VS since taking the direct limit is an exact functor.)

COROLlaRY 4.4. Let $M$ be a weight module with $V S 0=M_{0} \subset M_{1} \subset \ldots$, with factors $F_{i}=M_{i} / M_{i-1} \cong M\left(\lambda_{i}\right)$, and let $S \subseteq \mathfrak{h}^{*}$ such that $\lambda_{i} \in S$ for all $i$. Suppose $S=S_{1} \cup S_{2} \cup \cdots \cup S_{k}$ is a disjoint union such that $\operatorname{Hom}(M(\mu), M(\nu))=0$ whenever $\mu \in S_{i}$ and $\nu \in S_{j}$ with $i<j$. Then there exists a filtration $0=N_{0} \subset$ $N_{1} \subset \cdots \subset N_{k}=M$ such that each $N_{j} / N_{j-1}(j=1, \ldots, k)$ has a VS whose factors are precisely those $F_{i}$ with $\lambda_{i} \in S_{j}$.

PROOF. This follows from the proposition by an easy induction on $k$.

PROPOSITION 4.5. Let $M^{\prime}$ and $M^{\prime \prime}$ both satisfy the hypotheses of Corollary 4.4 with $S=S_{1} \cup \cdots \cup S_{k}$ a disjoint union as stated there. Let $0=N_{0}^{\prime} \subset N_{1}^{\prime} \subset$ $\cdots \subset N_{k}^{\prime}=M^{\prime}$ and $0=N_{0}^{\prime \prime} \subset N_{1}^{\prime \prime} \subset \cdots \subset N_{k}^{\prime \prime}=M^{\prime \prime}$ be filtrations as given in the 
conclusion of Corollary 4.4. Then for any $\mathfrak{g}$-module homomorphism $\phi: M^{\prime} \rightarrow M^{\prime \prime}$, we have $\phi\left(N_{j}^{\prime}\right) \subseteq N_{j}^{\prime \prime}$ for all $j=0,1, \ldots, k$, so that $\phi$ induces $\mathfrak{g}$-module maps $\phi_{j}: N_{j}^{\prime} / N_{j-1}^{\prime} \rightarrow N_{j}^{\prime \prime} / N_{j-1}^{\prime \prime}$.

Proof. If this did not hold for some $j$, then the composition $N_{j}^{\prime} \stackrel{\phi}{\rightarrow} M^{\prime \prime} \rightarrow$ $M^{\prime \prime} / N_{j}^{\prime \prime}$ would be nonzero, so that $\operatorname{Hom}(M(\mu), M(\nu))$ would be nonzero for some $\mu \in S_{1} \cup \cdots \cup S_{j}$ and some $\nu \in S_{j+1} \cup \cdots \cup S_{k}$, contrary to hypothesis.

COROLlaRY 4.6. Let $M$ and $S=S_{1} \cup \cdots \cup S_{k}$ satisfy the hypotheses of Corollary 4.4. Then the filtration $0=N_{0} \subset \cdots \subset N_{k}=M$ given in the conclusion of Corollary 4.4 is unique.

PROOF. Apply the proposition to any two such filtrations, taking $\phi: M \rightarrow M$ to be the identity map.

For the proof of the next theorem, we will need a technical fact regarding characters of Verma modules, which is easy to prove.

LEMMA 4.7 [9, LEMMA 5.3]. Let $\lambda \in \mathfrak{h}^{*}$, and suppose that $\sum_{\mu \leq \lambda} a_{\mu} \operatorname{ch} M(\mu)$ $=0$ for some set of integer coefficients $a_{\mu}$. Then $a_{\mu}=0$ for all $\mu \leq \bar{\lambda}$.

THEOREM 4.8. Let $\lambda \in \mathfrak{h}^{*}$, and let $S \subseteq\{\mu \mid \mu \leq \lambda\}$. Suppose

$$
0 \rightarrow M^{\prime} \stackrel{\phi}{\rightarrow} M \stackrel{\psi}{\rightarrow} M^{\prime \prime} \rightarrow 0
$$

is a short exact sequence in $C(\lambda)$. Further suppose that each of $M^{\prime}, M$, and $M^{\prime \prime}$, together with $S=S_{1} \cup \cdots \cup S_{k}$ satisfies the hypotheses of Corollary 4.4 . Let $0=$ $N_{0}^{\prime} \subset N_{1}^{\prime} \subset \cdots \subset N_{k}^{\prime}=M^{\prime}, 0=N_{0} \subset N_{1} \subset \cdots \subset N_{k}=M$, and $0=N_{0}^{\prime \prime} \subset N_{1}^{\prime \prime} \subset$ $\cdots \subset N_{k}^{\prime \prime}=M^{\prime \prime}$ be the filtrations given by Corollary 4.4. Then each of the induced sequences

$$
0 \rightarrow N_{j}^{\prime} / N_{j-1}^{\prime} \stackrel{\phi_{j}}{\rightarrow} N_{j} / N_{j-1} \stackrel{\psi_{j}}{\rightarrow} N_{j}^{\prime \prime} / N_{j-1}^{\prime \prime} \rightarrow 0
$$

is exact.

PROOF. We assume $k=2$, since for $k>2$, the result follows by induction from the case $k=2$.

First, consider

$$
0 \rightarrow N_{1}^{\prime} \stackrel{\phi_{1}}{\rightarrow} N_{1} \stackrel{\psi_{1}}{\rightarrow} N_{1}^{\prime \prime} \rightarrow 0
$$

Clearly $\phi_{1}$ is injective, being the restriction of $\phi$, and $\operatorname{Im} \phi_{1} \subseteq \operatorname{Ker} \psi_{1}$ for the same reason. Let $0=V_{0} \subset V_{1} \subset \cdots$ be a VS for $N_{1}^{\prime \prime}$, so that $N_{1}^{\prime \prime}=\bigcup_{i \geq 0} V_{i}$ and each $V_{i} / V_{i-1} \cong M\left(\mu_{i}\right)$ for some $\mu_{i} \in S_{1}$. For each $i=1,2, \ldots$, choose $v_{i} \in\left(V_{i}\right)_{\mu_{i}}$ so that $v_{i}+V_{i-1}$ is a highest weight vector for $V_{i} / V_{i-1}$. Note that $N_{1}^{\prime \prime}$ is generated by $\left\{v_{1}, v_{2}, \ldots\right\}$. Thus, if every $v_{i} \in \operatorname{Im} \psi_{1}$ then $\psi_{1}$ is surjective. To show that every $v_{i} \in \operatorname{Im} \psi_{1}$, suppose that some $v_{i} \notin \operatorname{Im} \psi_{1}$. We may choose such an $i$ to be minimal, so that $\left\{v_{1}, \ldots, v_{i-1}\right\} \subseteq \operatorname{Im} \psi_{1}$ and thus $V_{i-1} \subseteq \operatorname{Im} \psi_{1}$ since $\left\{v_{1}, \ldots, v_{i-1}\right\}$ generates $V_{i-1}$. But then we have a nontrivial homomorphism $M\left(\mu_{i}\right) \cong V_{i} / V_{i-1} \rightarrow$ $N_{1}^{\prime \prime} / \operatorname{Im} \psi_{1} \rightarrow M^{\prime \prime} / \operatorname{Im} \psi_{1}$ induced by the inclusion maps. Thus $\left(M^{\prime \prime} / \operatorname{Im} \psi_{1}\right.$ : $\left.L\left(\mu_{i}\right)\right) \neq 0$. But we also have an epimorphism $M / N_{1} \rightarrow M^{\prime \prime} / \operatorname{Im} \psi_{1}$ induced by $\psi$, so that any subquotient of $M^{\prime \prime} / \operatorname{Im} \psi_{1}$ is also a subquotient of $M / N_{1}$. This implies that $\left(M / N_{1}: L\left(\mu_{i}\right)\right) \neq 0$, and hence $\left(M(\mu): L\left(\mu_{i}\right)\right) \neq 0$ for some factor 
$M(\mu)$ in the VS for $M / N_{1}$, with $\mu \in S_{2}$. By Proposition 4.1, $\operatorname{Hom}\left(M\left(\mu_{i}\right), M(\mu)\right) \neq$ 0 . But this contradicts the assumption on $S_{1}$ and $S_{2}$, so we have that $v_{i} \in \operatorname{Im} \psi_{1}$ for all $i$, and hence $\operatorname{Im} \psi_{1}=N_{1}^{\prime \prime}$. This shows that

$$
\operatorname{ch} N_{1}=\operatorname{ch} N_{1}^{\prime}+\operatorname{ch} N_{1}^{\prime \prime}+\operatorname{ch}\left(\operatorname{Ker} \psi_{1} / \operatorname{Im} \phi_{1}\right) .
$$

On the other hand, we have

$$
\begin{aligned}
& \sum_{\mu \in S}(M: M(\mu)) \operatorname{ch} M(\mu)=\operatorname{ch} M=\operatorname{ch} M^{\prime}+\operatorname{ch} M^{\prime \prime} \\
& \quad=\sum_{\mu \in S}\left(M^{\prime}: M(\mu)\right) \operatorname{ch} M(\mu)+\sum_{\mu \in S}\left(M^{\prime \prime}: M(\mu)\right) \operatorname{ch} M(\mu) .
\end{aligned}
$$

By Lemma 4.7, $(M: M(\mu))=\left(M^{\prime}: M(\mu)\right)+\left(M^{\prime \prime}: M(\mu)\right)$ for each $\mu \in S$. Thus

$$
\begin{aligned}
\operatorname{ch} N_{1} & =\sum_{\mu \in S_{1}}(M: M(\mu)) \operatorname{ch} M(\mu) \\
& =\sum_{\mu \in S_{1}}\left(M^{\prime}: M(\mu)\right) \operatorname{ch} M(\mu)+\sum_{\mu \in S_{1}}\left(M^{\prime \prime}: M(\mu)\right) \operatorname{ch} M(\mu() \\
& =\operatorname{ch} N_{1}^{\prime}+\operatorname{ch} N_{1}^{\prime \prime},
\end{aligned}
$$

and

$$
\begin{aligned}
\operatorname{ch}\left(N_{2} / N_{1}\right) & =\sum_{\mu \in S_{2}}(M: M(\mu)) \operatorname{ch} M(\mu) \\
& =\sum_{\mu \in S_{2}}\left(M^{\prime}: M(\mu)\right) \operatorname{ch} M(\mu)+\sum_{\mu \in S_{2}}\left(M^{\prime \prime}: M(\mu)\right) \operatorname{ch} M(\mu) \\
& =\operatorname{ch}\left(N_{2}^{\prime} / N_{1}^{\prime}\right)+\operatorname{ch}\left(N_{2}^{\prime \prime} / N_{1}^{\prime \prime}\right) .
\end{aligned}
$$

Comparing (4.2) and (4.3) gives $\operatorname{ch}\left(\operatorname{Ker} \psi_{1} / \operatorname{Im} \phi_{1}\right)=0$, so that $\operatorname{Ker} \psi_{1}=\operatorname{Im} \phi_{1}$, and the sequence (4.1) is exact.

Now consider the sequence

$$
0 \rightarrow N_{2}^{\prime} / N_{1}^{\prime} \stackrel{\phi_{2}}{\rightarrow} N_{2} / N_{1} \stackrel{\psi_{2}}{\rightarrow} N_{2}^{\prime \prime} / N_{1}^{\prime \prime} \rightarrow 0
$$

Clearly $\operatorname{Im} \phi_{2} \subseteq \operatorname{Ker} \psi_{2}$, since $\psi_{2} \circ \phi_{2}$ is induced by $\psi \circ \phi$, and $\psi_{2}$ is surjective, since it is induced by $\psi$. Let $v+N_{1} \in \operatorname{Ker} \psi_{2}$. Then $\psi(v) \in N_{1}^{\prime \prime}=\operatorname{Im} \psi_{1}$, so that there exists $u \in N_{1}$ such that $\psi(u)=\psi(v)$. Thus $v-u \in \operatorname{Ker} \psi=\operatorname{Im} \phi$, and there exists $w \in M^{\prime}$ such that $\phi(w)=v-u$. But then $\phi_{2}\left(w+N_{1}^{\prime}\right)=\phi(w)+N_{1}=v-u+N_{1}=v+N_{1}$, and we see that $\operatorname{Ker} \psi_{2}=\operatorname{Im} \phi_{2}$. This shows that

$$
\operatorname{ch}\left(N_{2} / N_{1}\right)=\operatorname{ch}\left(N_{2}^{\prime} / N_{1}^{\prime}\right)+\operatorname{ch}\left(N_{2}^{\prime \prime} / N_{1}^{\prime \prime}\right)-\operatorname{ch}\left(\operatorname{Ker} \phi_{2}\right) .
$$

Comparing (4.4) with (4.6) gives $\operatorname{ch}\left(\operatorname{Ker} \phi_{2}\right)=0$, so that the sequence (4.5) is exact.

5. Definition of the functors $W_{k}^{\nu}$. We use the results of the previous two sections to define certain functors on $C(\lambda)$ and to show that these functors enjoy nice homological properties. We will use Weyl group orbits in $\mathfrak{h}^{*}$ to partition $S=\{\mu \mid \mu \leq \lambda\}$ as in the last section. We thus begin with some facts regarding Weyl group orbits.

The following proposition is well known. For a proof in the case of a nonsymmetric GCM, see [9, Lemma 4.9]. 
Proposition 5.1. Let $w \in W$ and $i \in\{1, \ldots, l\}$. If $\nu \in P^{+}$, then $r_{i} w \cdot \nu<w \cdot \nu$ if and only if $l\left(r_{i} w\right)>l(w)$. If $\nu+\rho \in P^{+}$, then $l\left(r_{i} w\right)>l(w)$ implies $r_{i} w \cdot \nu \leq w \cdot \nu$.

Observe that an easy induction on $l(w)$ gives $w \cdot \nu \leq \nu$ for all $w \in W$ when $\nu+\rho \in P^{+}$.

Proposition $5.2\left[4\right.$, Proposition 2.9]. Let $\nu \in P$. If there exists $\mu \in \mathfrak{h}^{*}$ such that $w \cdot \nu \leq \mu$ for all $w \in W$, then for some $w \in W$ we have $w \cdot \nu+\rho \in P^{+}$.

The next proposition is proved using the completion functors of T. Enright introduced in [3] and generalized to Kac-Moody algebras in [11].

Proposition 5.3 [11, Propositions 8.11 AND 8.13]. Let $\mu, \nu \in P$ and $i \in\{1, \ldots, l\}$. Let $\bar{\mu}$ be the higher of $\mu$ and $r_{i} \cdot \mu$, and let $\bar{\nu}$ be the higher of $\nu$ and $r_{i} \cdot \nu$. Then any imbedding of $M(\mu)$ in $M(\nu)$ extends to one of $M(\bar{\mu})$ in $M(\bar{\nu})$.

COROLlaRY 5.4. Let $\nu+\rho \in P^{+}$and $w \in W$. If $\operatorname{Hom}(M(\mu), M(w \cdot \nu)) \neq 0$, then there is some $w^{\prime} \in W$ with $w^{\prime} \cdot \mu+\rho \in P^{+}$and $\operatorname{Hom}\left(M\left(w^{\prime} \cdot \mu\right), M(\nu)\right) \neq 0$.

PROOF. It is well known, and easy to show by induction on $l(w)$, that $M(w \cdot \nu)$ imbeds in $M(\nu)$, so we have $\operatorname{Hom}(M(\mu), M(\nu)) \neq 0$. But now, by Proposition 5.3 and induction on $l(x), \operatorname{Hom}(M(x \cdot \mu), M(\nu)) \neq 0$ for all $x \in W$. In particular, $x \cdot \mu \leq \nu$ for all $x \in W$, and by Proposition 5.2, we may choose $w^{\prime} \in W$ such that $w^{\prime} \cdot \mu+\rho \in P^{+}$.

For the remainder of this section, fix $\lambda, \nu \in \mathfrak{h}^{*}$ such that $\nu \leq \lambda$ and $\nu+\rho \in P^{+}$. Take $S=\{\mu \mid \mu \leq \lambda\}, S_{1}=\{\mu \in S \mid w \cdot \mu \not \nu$ for some $w \in W\}, S_{2}=\{w \cdot \nu \mid w \in$ $W\}=W \cdot \nu$, and $S_{3}=\{\mu \in S \mid w \cdot \mu<\nu$ for all $w \in W\}$. Observe that $S$ is the disjoint union of $S_{1}, S_{2}$, and $S_{3}$.

LEMMA 5.5. Let $S_{1}, S_{2}$, and $S_{3}$ be as in the above discussion. Then

$$
\operatorname{Hom}(M(\mu), M(\chi))=0
$$

whenever $\mu \in S_{i}$ and $\chi \in S_{j}$ with $i<j$.

ProOF. We have three cases to consider. If $\mu \in S_{1}$ and $\chi \in S_{2}$, then $\chi=w \cdot \nu$ for some $w \in W$. Suppose $\operatorname{Hom}(M(\mu), M(\chi)) \neq 0$. Then, by Corollary 5.4, there is some $w^{\prime} \in W$ with $w^{\prime} \cdot \mu+\rho \in P^{+}$and $\operatorname{Hom}\left(M\left(w^{\prime} \cdot \mu\right), M(\nu)\right) \neq 0$. Thus $w^{\prime} \cdot \mu \leq \nu$, and by Proposition 5.1 and an easy induction on $l(x), x w^{\prime} \cdot \mu \leq \nu$ for all $x \in W$. This contradicts the definition of $S_{1}$, and therefore $\operatorname{Hom}(M(\mu), M(\chi))=0$.

For the other two cases, observe that if $\chi \in S_{3}$, then by Proposition 5.2 we must have $\chi=w \cdot \eta$ for some $\eta<\nu$ and $w \in W$ with $\eta+\rho \in P^{+}$. Thus, if $\mu \in S_{1}$ and $\chi \in S_{3}$, then the same argument as in the above paragraph shows $\operatorname{Hom}(M(\mu), M(\chi))=0$. Also, if $\mu \in S_{2}$ and $\chi \in S_{3}$ with $\operatorname{Hom}(M(\mu), M(\chi)) \neq 0$, then since $\chi=w \cdot \eta$ with $\eta+\rho \in P^{+}$we have by Corollary 5.4 that there exists $w^{\prime} \in$ $W$ with $w^{\prime} \cdot \mu+\rho \in P^{+}$and $\operatorname{Hom}\left(M\left(w^{\prime} \cdot \mu\right), M(\eta)\right) \neq 0$. But then $w^{\prime} \cdot \mu \leq \eta$, so that $x w^{\prime} \cdot \mu \leq \eta$ for all $x \in W$, by Proposition 5.1 and induction on $l(x)$. In particular, $\nu \leq \eta=w^{-1} \cdot \chi$, contradicting the fact that $\chi \in S_{3}$. Thus $\operatorname{Hom}(M(\mu), M(\chi))=0$ in this case, also.

The above lemma allows us to apply the results of $\S 4$ to any $M \in \mathrm{ObC}(\lambda)$ with a VS, obtaining a filtration $0=N_{0} \subset N_{1} \subset N_{2} \subset N_{3}=M$ as in Corollary 4.4. We will be particularly interested in the subquotient $N_{2} / N_{1}$ of $M$, which we 
henceforth denote $M^{(\nu)}$, so that $M^{(\nu)}$ has a VS whose factors are precisely those of $M$ with highest weights in the Weyl group orbit $W \cdot \nu$. Also, if $\phi: M \rightarrow M^{\prime}$ is a $\mathfrak{g}$-module homomorphism, we will denote the induced map $\phi_{2}: M^{(\nu)} \rightarrow M^{(\nu)}$ given in Proposition 4.5 by $\phi^{(\nu)}: M^{(\nu)} \rightarrow M^{\prime(\nu)}$. Recall that the finitely generated projectives in $C(\lambda)$ have finite VS, so that these results may be applied to them.

DEFINITION 5.6. Let $M \in \mathrm{Ob} C(\lambda), \mu \in \mathfrak{h}^{*}$, and suppose that

$$
\cdots \rightarrow P_{k} \stackrel{d_{k}}{\rightarrow} \cdots \stackrel{d_{1}}{\rightarrow} P_{0} \stackrel{d_{0}}{\rightarrow} M \rightarrow 0
$$

is a local projective resolution of $M$ at $\mu$. For each $k=0,1, \ldots$, we define the module $W_{k, \mu}^{\nu} M$ to be the $k$ th homology of the complex

$$
\stackrel{d_{k+1}^{(\nu)}}{\rightarrow} P_{k}^{(\nu)} \stackrel{d_{k}^{(\nu)}}{\rightarrow} \cdots \stackrel{d_{1}^{(\nu)}}{\rightarrow} P_{0}^{(\nu)} \rightarrow 0 .
$$

If $M^{\prime} \in \mathrm{Ob} C(\lambda)$ and

$$
\cdots \rightarrow Q_{k} \stackrel{d_{k}^{\prime}}{\rightarrow} \cdots \stackrel{d_{1}^{\prime}}{\rightarrow} Q_{0} \stackrel{d_{0}}{\rightarrow} M^{\prime} \rightarrow 0
$$

is also a local projective resolution at $\mu$, for any $\phi: M \rightarrow M^{\prime}$ we let

$$
\begin{array}{lllllllllll}
\cdots & \rightarrow & P_{k} & \stackrel{d_{k}}{\rightarrow} & \ldots & \stackrel{d_{1}}{\rightarrow} & P_{0} & \stackrel{d_{0}}{\rightarrow} & M & \rightarrow & 0 \\
& \downarrow \phi_{k} & & & & & \downarrow \phi_{0} & & \downarrow \phi & & \\
\cdots & \rightarrow & Q_{k} & \stackrel{d_{k}^{\prime}}{\rightarrow} & \ldots & \stackrel{d_{1}^{\prime}}{\rightarrow} & Q_{0} & \stackrel{d_{0}^{\prime}}{\rightarrow} & M^{\prime} & \rightarrow & 0
\end{array}
$$

be a morphism of complexes as in Proposition 3.8. Then we define $W_{k, \mu}^{\nu} \phi: W_{k, \mu}^{\nu} M \rightarrow$ $W_{k, \mu}^{\nu} M^{\prime}$ to be the map which $\phi_{k}^{(\nu)}$ induces in homology.

Observe that, since the morphism of complexes in Proposition 3.8 is unique up to homotopy, the map $W_{k, \mu}^{\nu} \phi$ is independent of the choice of the morphism of complexes. In fact, applying this argument to the identity map $M \rightarrow M$, we see that $W_{k, \mu}^{\nu} M$ is independent of the choice of the local projective resolution at $\mu$. It is now easy to see that $W_{k, \mu}^{\nu}$ is a functor on $C(\lambda)$.

REMARK. It appears that $W_{k, \mu}^{\nu} M$ may depend on $\lambda$, since the projectives in $C(\lambda)$ are most likely not projective in other such categories. We will see, however, in Corollary 6.4 below, that this is not the case, provided $\lambda$ is such that all weights of $M$ are $\leq \lambda$. For this reason, we have chosen to omit $\lambda$ from the already cumbersome notation for our functors.

By Proposition 3.8, if $M \in \mathrm{Ob} C(\lambda)$ and $\mu_{1} \geq \mu_{2}$, then for any local projective resolutions

$$
\cdots \rightarrow P_{k} \rightarrow \cdots \rightarrow P_{0} \rightarrow M \rightarrow 0
$$

and

$$
\cdots \rightarrow Q_{k} \rightarrow \cdots \rightarrow Q_{0} \rightarrow M \rightarrow 0
$$

of $M$ at $\mu_{1}$ and $\mu_{2}$, respectively, we obtain from the identity map $M \rightarrow M$ a morphism of complexes which induces a map in homology $W_{k, \mu_{1}}^{\nu} M \rightarrow W_{k, \mu_{2}}^{\nu} M$ which is well defined, since the morphism of complexes is unique up to homotopy. For the same reason, when $\mu_{1} \geq \mu_{2} \geq \mu_{3}$, the composition $W_{k, \mu_{1}}^{\nu} M \rightarrow W_{k, \mu_{2}}^{\nu} M \rightarrow$ $W_{k, \mu_{3}}^{\nu} M$ is the same as the map $W_{k, \mu_{1}}^{\nu} M \rightarrow W_{k, \mu_{3}}^{\nu} M$, since we may simply compose the two morphisms of complexes. This allows us to make the following definition. 
DEFinition 5.7. Let $M \in \mathrm{Ob} C(\lambda)$. For each $k=0,1, \ldots$, define $W_{k}^{\nu} M=$ inj $\lim W_{k, \mu}^{\nu} M$. Similarly, if $\phi: M \rightarrow M^{\prime}$ is a morphism in $C(\lambda)$, define $W_{k}^{\nu} \phi=$ inj $\lim W_{k, \mu}^{\nu} \phi$.

REMARK. For now, we have no guarantee that $W_{k}^{\nu} M$ is an object in $C(\lambda)$, since the weight spaces may no longer be finite dimensional. We will see in Corollary 6.3 below that it is an object in $C(\lambda)$, but for now, we simply consider $W_{k}^{\nu} M$ to be a g-module.

THEOREM 5.8. Let $\mu \in \mathfrak{h}^{*}$, and suppose

$$
0 \rightarrow M^{\prime} \stackrel{\phi}{\rightarrow} M \stackrel{\psi}{\rightarrow} M^{\prime \prime} \rightarrow 0
$$

is a short exact sequence in $C(\lambda)$. Then there is a long exact sequence

$$
\begin{aligned}
\cdots & \rightarrow W_{k, \mu}^{\nu} M^{\prime} \stackrel{W_{k, \mu}^{\nu} \phi}{\rightarrow} W_{k, \mu}^{\nu} M \stackrel{W_{k, \mu}^{\nu} \psi}{\rightarrow} W_{k, \mu}^{\nu} M^{\prime \prime} \\
& \rightarrow W_{k-1, \mu}^{\nu} M^{\prime} \stackrel{W_{k-1, \mu}^{\nu} \phi}{\rightarrow} \ldots \stackrel{W_{0, \mu}^{\nu} \psi}{\rightarrow} W_{0, \mu}^{\nu} M^{\prime \prime} \rightarrow 0 .
\end{aligned}
$$

PROOF. By Proposition 3.10 we may choose local projective resolutions

$$
\begin{aligned}
& \cdots \rightarrow P_{k}^{\prime} \rightarrow \cdots \rightarrow P_{0}^{\prime} \rightarrow M^{\prime} \rightarrow 0 \\
& \cdots \rightarrow P_{k} \rightarrow \cdots \rightarrow P_{0} \rightarrow M \rightarrow 0
\end{aligned}
$$

and

$$
\cdots \rightarrow P_{k}^{\prime \prime} \rightarrow \cdots \rightarrow P_{0}^{\prime \prime} \rightarrow M^{\prime \prime} \rightarrow 0
$$

of $M^{\prime}, M$, and $M^{\prime \prime}$, respectively, at $\mu$ in such a way that

$$
\begin{aligned}
& \cdots \rightarrow \begin{array}{ccc}
0 \\
\downarrow \\
P_{k}^{\prime} \\
\downarrow \phi_{k}
\end{array} \rightarrow \cdots \rightarrow \begin{array}{c}
0 \\
P_{0}^{\prime} \\
P_{k}
\end{array} \rightarrow 0 \\
& \cdots \quad \rightarrow P_{k} \rightarrow \cdots \rightarrow P_{0} \rightarrow 0 \\
& \cdots \rightarrow \begin{array}{c}
\downarrow \psi_{k} \\
P_{k}^{\prime \prime} \\
\downarrow
\end{array} \rightarrow \cdots \rightarrow \begin{array}{c} 
\\
\downarrow \\
0
\end{array}
\end{aligned}
$$

is a short exact sequence of complexes. Note that each column is a short exact sequence of modules with VS, so that passing to the subquotients,

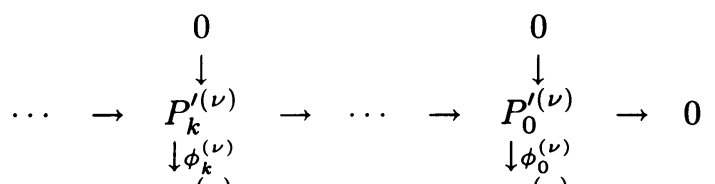

$$
\begin{aligned}
& \cdots \rightarrow \begin{array}{l}
P_{k}^{(\nu)} \\
\downarrow \psi_{k}^{(\nu)}
\end{array} \rightarrow \cdots \rightarrow \begin{array}{l}
P_{0}^{(\nu)} \\
\downarrow \psi_{0}^{(\nu)}
\end{array} \rightarrow 0
\end{aligned}
$$

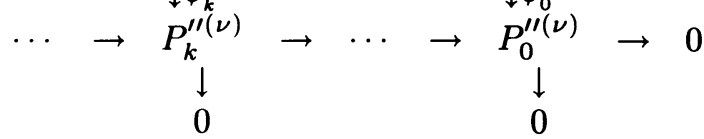

is a short exact sequence of complexes by Theorem 4.8. Therefore, we obtain the long exact sequence in homology. 
COROLlary 5.9. Suppose

$$
0 \rightarrow M^{\prime} \stackrel{\phi}{\rightarrow} M \stackrel{\psi}{\rightarrow} M^{\prime \prime} \rightarrow 0
$$

is a short exact sequence in $C(\lambda)$. Then there is a long exact sequence

$$
\begin{aligned}
\cdots & \rightarrow W_{k}^{\nu} M^{\prime} \stackrel{W_{k}^{\nu} \phi}{\rightarrow} W_{k}^{\nu} M \stackrel{W_{k}^{\nu} \psi}{\rightarrow} W_{k}^{\nu} M^{\prime \prime} \\
& \rightarrow W_{k-1}^{\nu} M^{\prime} \stackrel{W_{k-1}^{\nu} \phi}{\rightarrow} \ldots \stackrel{W_{0}^{\nu} \psi}{\rightarrow} W_{0}^{\nu} M^{\prime \prime} \rightarrow 0 .
\end{aligned}
$$

PrOOF. This follows from the theorem and the fact that taking the direct limit preserves exactness.

6. Properties of the functors $W_{k}^{\nu}$. We retain the notation of $\S 5$. We will analyze in greater detail the modules $W_{k}^{\nu} M$ for the case where $M$ has a VS, and use this analysis to prove certain properties of the functors $W_{k}^{\nu}$.

Proposition 6.1. Let $M \in \mathrm{Ob} C(\lambda)$ have a VS. Then

$$
W_{k}^{\nu} M \cong \begin{cases}M^{(\nu)} & \text { if } k=0, \\ 0 & \text { if } k>0 .\end{cases}
$$

Furthermore, if $M$ and $N$ are two such modules, and $\phi: M \rightarrow N$ is a $\mathfrak{g}$-module map, then $W_{0}^{\nu} \phi$ is just the map $\phi^{(\nu)}$ induced by $\phi$.

ProOF. We first consider the functors $W_{k, \mu}^{\nu}$ for $\mu \in \mathfrak{h}^{*}$. Suppose that $0=$ $M_{0} \subset M_{1} \subset \cdots$ and $0=N_{0} \subset N_{1} \subset \cdots$ are VS for $M$ and $N$, respectively. By Proposition 4.1 and Lemma 4.2, we may alter the VS, if necessary, so that for some $m, n \in \mathbf{Z}_{+}$we have $0=M_{0} \subset M_{1} \subset \cdots \subset M_{m}$ and $0=N_{0} \subset N_{1} \subset \cdots \subset N_{n}$ in which all Verma factors are of the form $M(\chi)$ with $\chi \geq \mu$ and $M / M_{m}$ and $N / N_{n}$ have no weights $\geq \mu$. Observe that $\phi\left(M_{m}\right) \subseteq N_{n}$, since $M_{m}$ is generated by vectors of weights $\geq \mu$. Now, applying Theorem 3.5 to $M_{m}$ and $N_{n}$, we obtain projective resolutions

$$
\cdots \rightarrow P_{k} \stackrel{d_{k}}{\rightarrow} \cdots \rightarrow P_{0} \stackrel{d_{0}}{\rightarrow} M_{m} \rightarrow 0
$$

and

$$
\cdots \rightarrow Q_{k} \stackrel{d_{k}^{\prime}}{\rightarrow} \cdots \rightarrow Q_{0} \stackrel{d_{0}^{\prime}}{\rightarrow} N_{n} \rightarrow 0
$$

such that all kernels and images have VS, and where each $P_{k}$ and each $Q_{k}$ is a finite direct sum of various $I(\chi)$ with $\chi \geq \mu$. Note that the restricted map $\phi: M_{m} \rightarrow N_{n}$ induces maps $\phi_{k}: P_{k} \rightarrow Q_{k}$ for $k=0,1, \ldots$ in the usual way to give a morphism of complexes. Since $M / M_{m}$ and $N / N_{n}$ have no weights $\geq \mu$, these resolutions (followed by the inclusion maps $M_{m} \subseteq M$ and $N_{n} \subseteq N$ ) are actually local projective resolutions of $M$ and $N$ at $\mu$.

Now, since all the kernels and images in (6.1) and (6.2) have VS, these resolutions are obtained by splicing (i.e taking the Yoneda product of) short exact sequences in which all terms have VS, so that by Theorem 4.8 the commutative diagram

$$
\begin{array}{lllllllllll}
\cdots & \rightarrow & P_{k}^{(\nu)} & \stackrel{d_{k}^{(\nu)}}{\rightarrow} & \cdots & \rightarrow & P_{0}^{(\nu)} & \stackrel{d_{0}^{(\nu)}}{\rightarrow} & M_{m}^{(\nu)} & \rightarrow & 0 \\
& \downarrow \phi_{k}^{(\nu)} & & & & & & \\
\cdots & (\nu) & & \downarrow_{\phi}^{(\nu)} & & \\
\cdots & \rightarrow & Q_{k}^{(\nu)} & \stackrel{d_{k}^{\prime(\nu)}}{\rightarrow} & \cdots & \rightarrow & Q_{0}^{(\nu)} & \stackrel{d_{0}^{(\nu)}}{\rightarrow} & N_{n}^{(\nu)} & \rightarrow & 0
\end{array}
$$


has exact rows. Thus, dropping the last module in each row of (6.3) and taking the homology gives $W_{k, \mu}^{\nu} M=0=W_{k, \mu}^{\nu} N$ for all $k>0, W_{0, \mu}^{\nu} M \cong M_{m}^{(\nu)}$, and $W_{0, \mu}^{\nu} N \cong N_{n}^{(\nu)}$ with the map $W_{0, \mu}^{\nu} \phi: M_{m}^{(\nu)} \rightarrow N_{n}^{(\nu)}$ being the induced map $\phi^{(\nu)}$. The result now follows by taking direct limits.

THEOREM 6.2. Let $M \in \mathrm{Ob} C(\lambda)$, and suppose

$$
\cdots \rightarrow D_{k} \stackrel{d_{k}}{\rightarrow} \ldots \stackrel{d_{1}}{\rightarrow} D_{0} \stackrel{d_{0}}{\rightarrow} M \rightarrow 0
$$

is an exact resolution of $M$ in $C(\lambda)$ in which every $D_{k}$ has a VS. Then $W_{k}^{\nu} M=$ $H_{k}\left(D_{*}^{(\nu)}\right)$. In other words, $W_{k}^{\nu} M$ may be computed as the kth homology of the complex

$$
\cdots \rightarrow D_{k}^{(\nu)} \stackrel{d_{k}^{(\nu)}}{\rightarrow} \cdots \stackrel{d_{1}^{(\nu)}}{\rightarrow} D_{0}^{(\nu)} \rightarrow 0 .
$$

PROOF. Let $N_{i}=\operatorname{Ker} d_{i}=\operatorname{Im} d_{i+1}$ for $i=0,1, \ldots$ and $N_{-1}=M$. Then we have short exact sequences

$$
0 \rightarrow N_{i} \stackrel{\phi_{i}}{\rightarrow} D_{i} \stackrel{\psi_{i}}{\rightarrow} N_{i-1} \rightarrow 0
$$

for all $i \geq 0$, where each $\phi_{i}$ is the inclusion map, and each $\psi_{i}$ is the restriction of $d_{i}$. Note that the composition

$$
W_{0}^{\nu} D_{i+1} \stackrel{W_{0}^{\nu} \psi_{i+1}}{\rightarrow} W_{0}^{\nu} N_{i} \stackrel{W_{0}^{\nu} \phi_{i}}{\rightarrow} W_{0}^{\nu} D_{i}
$$

is just the induced map

$$
D_{i+1}^{(\nu)} \stackrel{d_{i+1}^{(\nu)}}{\rightarrow} D_{i}^{(\nu)}
$$

by Proposition 6.1, so that $\operatorname{Im}\left(W_{0}^{\nu} \phi_{i}\right)=\operatorname{Im}\left(d_{i+1}^{(\nu)}\right)$. From the fact that $W_{1}^{\nu} D_{i}=0$ for all $i$ by Proposition 6.1, and from the long exact sequences in homology, we obtain the commutative diagram

$$
\begin{aligned}
& W_{0}^{\nu} N_{i+1} \\
& \downarrow W_{0}^{\nu} \phi_{i+1} \\
& W_{0}^{\nu} D_{i+1} \\
& 0 \rightarrow W_{1}^{\nu} N_{i-1} \rightarrow W_{0}^{\nu} N_{i} \underset{W_{0}^{\nu} \phi_{i}}{\stackrel{\downarrow W_{0}^{\nu} \psi_{i+1}}{d_{i+1}^{(\nu)}}} W_{0}^{\nu} D_{i} \underset{W_{0}^{\nu} \psi_{i}}{\overrightarrow{W_{0}}} W_{0}^{\nu} N_{i-1} \rightarrow 0 \\
& \downarrow
\end{aligned}
$$

with both the row and the column exact. Thus,

$$
\begin{aligned}
W_{1}^{\nu} N_{i-1} & \cong \operatorname{Ker} W_{0}^{\nu} \phi_{i} \cong \operatorname{Ker} d_{i+1}^{(\nu)} / \operatorname{Im} W_{0}^{\nu} \phi_{i+1} \\
& =\operatorname{Ker} d_{i+1}^{(\nu)} / \operatorname{Im} d_{i+2}^{(\nu)}=H_{i+1}\left(D_{*}^{(\nu)}\right)
\end{aligned}
$$

and $W_{0}^{\nu} N_{i-1} \cong \operatorname{Coker} W_{0}^{\nu} \phi_{i}=\operatorname{Coker} d_{i+1}^{(\nu)}$ for all $i \geq 0$. This gives immediately that $W_{0}^{\nu} M=W_{0}^{\nu} N_{-1} \cong \operatorname{Coker} d_{1}^{(\nu)}=H_{0}\left(D_{*}^{(\nu)}\right)$ and $W_{1}^{\nu} M=W_{1}^{\nu} N_{-1} \cong H_{1}\left(D_{*}^{(\nu)}\right)$. For $k>1$, we use the short exact sequence

$$
0 \rightarrow N_{0} \rightarrow D_{0} \rightarrow M \rightarrow 0
$$


to obtain the long exact homology sequence

$$
\cdots \rightarrow 0=W_{k}^{\nu} D_{0} \rightarrow W_{k}^{\nu} M \rightarrow W_{k-1}^{\nu} N_{0} \rightarrow W_{k-1}^{\nu} D_{0}=0 \rightarrow \cdots
$$

by Proposition 6.1. Thus $W_{k}^{\nu} M \cong W_{k-1}^{\nu} N_{0}$. By induction on $k$, since

$$
\cdots \rightarrow D_{k} \rightarrow \cdots \rightarrow D_{1} \rightarrow N_{0} \rightarrow 0
$$

is an exact resolution of $N_{0}$, we have $W_{k}^{\nu} M \cong W_{k-1}^{\nu} N_{0} \cong H_{k}\left(D_{*}^{(\nu)}\right)$.

We now use this theorem to obtain some properties of the functors $W_{k}^{\nu}$. For the proofs of these, recall the standard resolution

$$
\cdots \rightarrow U(\mathfrak{g}) \otimes_{U(\mathfrak{b})} \Lambda^{k}(\mathfrak{g} / \mathfrak{b}) \rightarrow \cdots \rightarrow U(\mathfrak{g}) \otimes_{U(\mathfrak{b})} \Lambda^{0}(\mathfrak{g} / \mathfrak{b}) \rightarrow K \rightarrow 0
$$

of the trivial one-dimensional module $K$ constructed and shown to be exact in $[\mathbf{1}$, Theorem II.9.1].

COROllary 6.3. For any $M \in \mathrm{Ob} C(\lambda)$ and any $k \geq 0, W_{k}^{\nu} M \in \mathrm{Ob} C(\lambda)$.

Proof. Tensoring the standard resolution of $K$ with $M$ and applying [4, Proposition 1.7] gives an exact resolution

$$
\cdots \rightarrow D_{k} \rightarrow \cdots \rightarrow D_{0} \rightarrow M \rightarrow 0
$$

of $M$ in $C(\lambda)$ with each $D_{k}=U(\mathfrak{g}) \otimes_{U(\mathfrak{b})}\left(\Lambda^{k}(\mathfrak{g} / \mathfrak{b}) \otimes_{K} M\right)$ for $k=0,1, \ldots$ It is well known, and easy to show, that each of the modules $D_{k}$ has a VS. Thus, by the theorem, $W_{k}^{\nu} M$ is the $k$ th homology of a complex in $C(\lambda)$, hence $W_{k}^{\nu} M$ is an object in $C(\lambda)$.

COROLlaRY 6.4. $W_{k}^{\nu} M$ is independent of the choice of $\lambda$, provided that all the weights of $M$ are $\leq \lambda$.

ProOF. Again, we consider the exact resolution (6.4) of $M$. The highest weights of the Verma factors in the VS for $D_{k}$ are just the weights of $\Lambda^{k}(\mathfrak{g} / \mathfrak{b}) \otimes_{K} M$, hence are all $\leq \lambda$ for any such $\lambda$. Thus, this resolution consists of modules in $C(\lambda)$ for any such $\lambda$, and the result now follows from Theorem 6.2.

COROllaRY 6.5. Let $\mu \in \mathfrak{g}^{*}$ and suppose $M \in \mathrm{Ob} C(\lambda)$ has no weights $\geq \mu$. Then $W_{k}^{\nu} M$ also has no weights $\geq \mu$ for each $k \geq 0$.

PROOF. This also follows from the resolution (6.4) of $M$, since none of the $D_{k}$ have any weights $\geq \mu$.

We have seen, from the long exact homology sequence, that $W_{0}^{\nu}$ is a right exact functor. In the remainder of this section, we will show that for certain "nice" modules, $W_{0}^{\nu}$ is "exact on the $W$-orbit of $\nu$," in the sense that the obstruction to its exactness involves only irreducible subquotients with highest weights outside of $W \cdot \nu$ (cf. Theorem 6.9 below for the precise statement).

For the following lemma, recall the sets $S=\{\mu \mid \mu \leq \lambda\}, S_{1}=\{\mu \mid w \cdot \mu \nsubseteq \nu$ for some $w \in W\}, S_{2}=W \cdot \nu$, and $S_{3}=\{\mu \mid w \cdot \mu<\nu$ for all $w \in W\}$ defined in $\S 5$.

LEMMA 6.6. Let $M \in \mathrm{Ob} C(\lambda)$ be a highest weight module of weight $\mu$. If $\mu \notin W \cdot \nu$, then $W_{0}^{\nu} M=0$. If $\mu \in W \cdot \nu$, then $W_{0}^{\nu} M$ is a nonzero highest weight module of weight $\mu$, and there is an epimorphism $\pi: W_{0}^{\nu} M \rightarrow M$ such that any irreducible subquotient of $\operatorname{Ker} \pi$ is of the form $L(\chi)$ with $\chi \in S_{3}$.

ProOF. Let

$$
0 \rightarrow V \rightarrow M(\mu) \rightarrow M \rightarrow 0
$$


be a short exact sequence. If $\mu \notin W \cdot \nu$, then by Proposition 6.1 we have $W_{0}^{\nu} M(\mu)=$ 0 , and since $W_{0}^{\nu}$ is right exact, $W_{0}^{\nu} M=0$. Thus, we may assumee that $\mu \in W \cdot \nu$.

Let

$$
\cdots \rightarrow V_{2} \rightarrow V_{1} \rightarrow V \rightarrow 0
$$

be an exact resolution of $V$ such that each $V_{n}$ has a VS. (One may be obtained by tensoring $V$ with the standard resolution of $K$.) Splicing together (6.5) and (6.6) gives the resolution

$$
\cdots \rightarrow V_{2} \rightarrow V_{1} \stackrel{\phi}{\rightarrow} M(\mu) \rightarrow M \rightarrow 0
$$

of $M$.

Recall the filtration $0=N_{0} \subset N_{1} \subset N_{2} \subset N_{3}=V_{1}$ of $V_{1}$ associated with the disjoint union $S=S_{1} \cup S_{2} \cup S_{3}$ given by Corollary 4.4, so that $V_{1}^{(\nu)}=N_{2} / N_{1}$. Now $\phi\left(N_{1}\right)=0$ by Lemma 5.5 , so that $\operatorname{Im} \phi^{(\nu)}=\phi\left(N_{2}\right) \subseteq V \subsetneq M(\mu)$. Now, by Theorem 6.2, $W_{0}^{\nu} M \cong \operatorname{Coker} \phi^{(\nu)}=M(\mu) / \operatorname{Im} \phi^{(\nu)}=M(\mu) / \phi\left(N_{2}\right)$. Thus, $W_{0}^{\nu} M$ is a nonzero quotient of $M(\mu)$, hence a nonzero highest weight module of weight $\mu$.

Also, from (6.7) we have $M \cong \operatorname{Coker} \phi=M(\mu) / \phi\left(N_{3}\right)$, so that there is an epimorphism $\pi: W_{0}^{\nu} M \cong M(\mu) / \phi\left(N_{2}\right) \rightarrow M(\mu) / \phi\left(N_{3}\right) \cong M$ induced by the identity map on $M(\mu)$. Note that $\operatorname{Ker} \pi \cong \phi\left(N_{3}\right) / \phi\left(N_{2}\right)$. Hence, any irreducible subquotient $L(\chi)$ of $\operatorname{Ker} \pi$ is a subquotient of $N_{3} / N_{2}$, and must therefore be a subquotient of some Verma module $M(\eta)$ with $\eta \in S_{3}$. By Proposition 4.1, $\operatorname{Hom}(M(\chi), M(\eta)) \neq 0$, and thus by Lemma 5.5 we must have $\chi \in S_{3}$.

Proposition 6.7. Let $M \in \mathrm{Ob} C(\lambda)$. For any $w \in W,\left(W_{0}^{\nu} M: L(w \cdot \nu)\right) \leq$ $(M: L(w \cdot \nu))$.

Proof. Let $0=M_{0} \subset M_{1} \subset \cdots \subset M_{n}=M$ be an LCS for $M$ at $w \cdot \nu$. We use induction on $n$.

If $n=0$, there is nothing to prove, so assume $n>0$. From the short exact sequence

$$
0 \rightarrow M_{n-1} \rightarrow M \rightarrow M / M_{n-1} \rightarrow 0
$$

we obtain the long exact sequence

$$
\cdots \rightarrow W_{0}^{\nu} M_{n-1} \rightarrow W_{0}^{\nu} M \rightarrow W_{0}^{\nu}\left(M / M_{n-1}\right) \rightarrow 0,
$$

which shows that

$$
\left(W_{0}^{\nu} M: L(w \cdot \nu)\right) \leq\left(W_{0}^{\nu} M_{n-1}: L(w \cdot \nu)\right)+\left(W_{0}^{\nu}\left(M / M_{n-1}\right): L(w \cdot \nu)\right) .
$$

By induction, $\left(W_{0}^{\nu} M_{n-1}: L(w \cdot \nu)\right) \leq\left(M_{n-1}: L(w \cdot \nu)\right)$. If $M / M_{n-1}$ has no weights $\geq w \cdot \nu$, then by Corollary 6.5 neither does $W_{0}^{\nu}\left(M / M_{n-1}\right)$, so we are done in this case. Otherwise, $M / M_{n-1} \cong L(\mu)$ for some $\mu \geq w \cdot \nu$. But then, by Lemma 6.6, the only way $L(w \cdot \nu)$ could occur as a subquotient of $W_{0}^{\nu} L(\mu)$ is to have $\mu=w \cdot \nu$, in which case it occurs with multiplicity one.

COROLlaRY 6.8. For any $\mu \in S_{2} \cup S_{3}$ and any $w \in W,\left(W_{1}^{\nu} L(\mu): L(w \cdot \nu)\right)=0$.

PROOF. Consider a short exact sequence

$$
0 \rightarrow M \rightarrow M(\mu) \rightarrow L(\mu) \rightarrow 0 .
$$


Since $W_{1}^{\nu} M(\mu)=0$ by Proposition 6.1 , the long exact homology sequence gives the exact sequence

$$
0 \rightarrow W_{1}^{\nu} L(\mu) \rightarrow W_{0}^{\nu} M \rightarrow W_{0}^{\nu} M(\mu) \rightarrow W_{0}^{\nu} L(\mu) \rightarrow 0 .
$$

This implies

$\left(W_{1}^{\nu} L(\mu): L(w \cdot \nu)\right)=\left(W_{0}^{\nu} M: L(w \cdot \nu)\right)-\left(W_{0}^{\nu} M(\mu): L(w \cdot \nu)\right)+\left(W_{0}^{\nu} L(\mu): L(w \cdot \nu)\right)$.

If $\mu \in S_{3}$, then $W_{0}^{\nu} M(\mu)=0=W_{0}^{\nu} L(\mu)$, so that

$$
\left(W_{1}^{\nu} L(\mu): L(w \cdot \nu)\right)=\left(W_{0}^{\nu} M: L(w \cdot \nu)\right) \leq(M: L(w \cdot \nu))
$$

by Proposition 6.7. But $L(w \cdot \nu)$ cannot be a subquotient of $M$ in this case, since otherwise it would be a subquotient of $M(\mu)$, so that by Proposition 4.1 we would have $\operatorname{Hom}(M(w \cdot \nu), M(\mu)) \neq 0$, contradicting Lemma 5.5. Thus $\left(W_{1}^{\nu} L(\mu)\right.$ : $L(w \cdot \nu))=0$ in case $\mu \in S_{3}$.

If $\mu \in S_{2}$, then $W_{0}^{\nu} M(\mu) \cong M(\mu)$ by Proposition 6.1, and there is an epimorphism $\pi: W_{0}^{\nu} L(\mu) \rightarrow L(\mu)$ with $(\operatorname{Ker} \pi: L(w \cdot \nu))=0$ by Lemma 6.6. Thus $\left(W_{0}^{\nu} M(\mu): L(w \cdot \nu)\right)=(M(\mu): L(w \cdot \nu))$ and $\left(W_{0}^{\nu} L(\mu): L(w \cdot \nu)\right)=(L(\mu): L(w \cdot \nu))$, and from Proposition 6.7 we obtain

$$
\begin{aligned}
& \left(W_{1}^{\nu} L(\mu): L(w \cdot \nu)\right) \\
& \quad=\left(W_{0}^{\nu} M: L(w \cdot \nu)\right)-\left(W_{0}^{\nu} M(\mu): L(w \cdot \nu)\right)+\left(W_{0}^{\nu} L(\mu): L(w \cdot \nu)\right) \\
& \quad=\left(W_{0}^{\nu} M: L(w \cdot \nu)\right)-(M(\mu): L(w \cdot \nu))+(L(\mu): L(w \cdot \mu)) \\
& \quad \leq(M: L(w \cdot \nu))-(M(\mu): L(w \cdot \nu))+(L(\mu): L(w \cdot \nu)) \\
& \quad=0,
\end{aligned}
$$

where we have used (6.8) for the last equality, and the result follows.

The next theorem deals with certain modules $M$ in the category $C(\lambda)$ which are "nice" with respect to $\nu$. We remark that the full subcategory of $C(\lambda)$ whose objects are those modules $M$ satisfying the hypotheses of the theorem is an abelian category, since it is closed under taking submodules, quotients, and finite direct sums.

THEOREM 6.9. Let $M \in \mathrm{Ob} C(\lambda)$ such that every irreducible subquotient of $M$ is of the form $L(\chi)$ with $\chi \in S_{2} \cup S_{3}$. Then for all $w \in W$ we have $\left(W_{0}^{\nu} M: L(w \cdot \nu)\right)=(M: L(w \cdot \nu))$ and $\left(W_{k}^{\nu} M: L(w \cdot \nu)\right)=0$ for all $k>0$.

ProOF. Let $0=M_{0} \subset M_{1} \subset \cdots \subset M_{n}=M$ be an LCS for $M$ at $w \cdot \nu$. We first consider $W_{0}^{\nu} M$, using induction on $n$. If $n=0$, then there is nothing to prove, so assume $n>0$, and consider the short exact sequence

$$
0 \rightarrow M_{n-1} \rightarrow M \rightarrow M / M_{n-1} \rightarrow 0 \text {. }
$$

This gives rise to the exact sequence

$$
\cdots \rightarrow W_{1}^{\nu}\left(M / M_{n-1}\right) \rightarrow W_{0}^{\nu} M_{n-1} \rightarrow W_{0}^{\nu} M \rightarrow W_{0}^{\nu}\left(M / M_{n-1}\right) \rightarrow 0 .
$$

Since $M / M_{n-1}$ either has no weights $\geq w \cdot \nu$ or is irreducible,

$$
\left(W_{1}^{\nu}\left(M / M_{n-1}\right): L(w \cdot \nu)\right)=0
$$

by Corollary 6.5 and Corollary 6.8. Thus

$$
\left(W_{0}^{\nu} M: L(w \cdot \nu)\right)=\left(W_{0}^{\nu} M_{n-1}: L(w \cdot \nu)\right)+\left(W_{0}^{\nu}\left(M / M_{n-1}\right): L(w \cdot \nu)\right),
$$


and by induction and Lemma 6.6 we are done with the proof of the statement regarding $W_{0}^{\nu} M$.

Now, for $k=1$ we have that $\left(W_{1}^{\nu} F: L(w \cdot \nu)\right)=0$ when $F$ is a factor in the LCS for $M$ at $w \cdot \nu$, by Corollaries 6.5 and 6.8. Thus, from the exact sequence

$$
W_{1}^{\nu} M_{n-1} \rightarrow W_{1}^{\nu} M \rightarrow W_{1}^{\nu}\left(M / M_{n-1}\right),
$$

we have $\left(W_{1}^{\nu} M: L(w \cdot \nu)\right)=0$ by induction on the number of factors in the LCS.

For $k>1$, we use induction on $k$. First consider $L(\chi)$ for $\chi \in S_{2} \cup S_{3}$. From the short exact sequence

$$
0 \rightarrow N \rightarrow M(\chi) \rightarrow L(\chi) \rightarrow 0
$$

and Proposition 6.1, we obtain the exact sequence

$$
0=W_{k}^{\nu} M(\chi) \rightarrow W_{k}^{\nu} L(\chi) \rightarrow W_{k-1}^{\nu} N \rightarrow W_{k-1}^{\nu} M(\chi)=0,
$$

so that $\left(W_{k}^{\nu} L(\chi): L(w \cdot \nu)\right)=\left(W_{k-1}^{\nu} N: L(w \cdot \nu)\right)$. Note that by Proposition 4.1 and Lemma 5.5, $N$ satisfies the hypotheses of the theorem, so that by induction on $k,\left(W_{k}^{\nu} L(\chi): L(w \cdot \nu)\right)=\left(W_{k-1}^{\nu} N: L(w \cdot \nu)\right)=0$. Finally, this fact together with Corollary 6.5 shows that any factor $F$ in the LCS for $M$ at $w \cdot \nu$ satisfies $\left(W_{k}^{\nu} F: L(w \cdot \nu)\right)=0$, so that an easy induction on the number of factors gives $\left(W_{k}^{\nu} M: L(w \cdot \nu)\right)=0$.

7. Translation functors. In this section we use the functors $W_{0}^{\nu}$ to define translation functors analogous to those introduced by J. C. Jantzen in [5] and, applying these to Verma modules and irreducibles, we obtain a translation principle regarding multiplicities of irreducible subquotients in Verma modules.

We will place some restrictions on $\lambda$ and $\nu$ henceforth. In particular, assume $\lambda \in P^{+}$. Let $\eta \in P^{+}$, and set $\nu=\lambda+\eta$. Observe that this implies $\nu \in P^{+}$. Also, since $\eta \in P^{+}, L(\eta)$ is a locally finite $\mathfrak{a}_{i}$-module for each $i=1, \ldots, l$, so that by the representation theory of $s l(2, K), \operatorname{dim} L(\eta)_{\chi}=\operatorname{dim} L(\eta)_{r_{i} \chi}$ for all $\chi \in \mathfrak{h}^{*}$ and all $i=1, \ldots, l$. By induction on $l(w), \operatorname{dim} L(\eta)_{\chi}=\operatorname{dim} L(\eta)_{w \chi}$ for all $\chi \in \mathfrak{h}^{*}$ and all $w \in W$.

DEFinition 7.1. For any $M \in \mathrm{Ob} C(\lambda)$, define the translation of $M$ from $\lambda$ to $\nu$ by $T_{\lambda}^{\nu} M=W_{0}^{\nu}\left(L(\eta) \otimes_{K} M\right)$. Thus, the functor $T_{\lambda}^{\nu}$ is the composition of the exact functor $L(\eta) \otimes_{K}-$ and the right exact functor $W_{0}^{\nu}$, so that $T_{\lambda}^{\nu}$ is a right exact functor on $C(\lambda)$.

Proposition 7.2. Let $\mu+\rho \in P^{+}$with $\mu<\lambda$, and let $w \in W$. Then $L(\eta) \otimes_{K}$ $M(w \cdot \mu)$ has a VS whose factors are of the form $M\left(w^{\prime} \cdot \chi\right)$ with $\chi<\nu$ and $\chi+\rho \in P^{+}$. Also, $L(\eta) \otimes_{K} M(w \cdot \lambda)$ has a VS whose factors are $M(w \cdot \nu)$ with multiplicity one together with factors of the form $M\left(w^{\prime} \cdot \chi\right)$ with $\chi<\nu$ and $\chi+\rho \in P^{+}$.

ProOF. In general, $L(\eta) \otimes_{K} M(\xi)$ has a VS with $\left(L(\eta) \otimes_{K} M(\xi): M(\varsigma)\right)=$ $\operatorname{dim} L(\eta)_{\zeta-\xi}$ for any $\xi, \varsigma \in \mathfrak{h}^{*}$. Thus, if $\mu \leq \lambda$ with $\mu+\rho \in P^{+}$, then for any Verma factor $M(\varsigma)$ in the VS for $L(\eta) \otimes_{K} M(w \cdot \mu)$ we have that $\varsigma-w \cdot \mu$ is a weight of $L(\eta)$. By the discussion preceding Definition 7.1, we see that $x(\varsigma-w \cdot \mu) \leq \eta$ for all $x \in W$. Also, by Proposition $5.1,(x w) \cdot \mu \leq \mu$ for all $x \in W$. Thus, for all $x \in W$ we have

$$
x \cdot \zeta=x \cdot(w \cdot \mu+\varsigma-w \cdot \mu)=(x w) \cdot \mu+x(\varsigma-w \cdot \mu) \leq \mu+\eta \leq \lambda+\eta=\nu .
$$


Note that equality can only hold in case $\mu=\lambda,(x w) \cdot \lambda=\lambda$, and $x(\varsigma-w \cdot \lambda)=\lambda$. Thus, in case $\mu<\lambda$ we have $x \cdot \zeta<\nu$ for all $x \in W$, so that by Proposition 5.2 , we have $\varsigma=w^{\prime} \cdot \chi$ for some $\chi<\eta$ with $\chi+\rho \in P^{+}$. In case $\mu=\lambda$, we still have $x \cdot \varsigma \leq \nu$ for all $x \in W$, with equality holding only when $(x w) \cdot \lambda=\lambda$ and $x(\varsigma-w \cdot \lambda)=\eta$. Thus, if $x \cdot \varsigma=\nu$ holds for some $x \in W$, then $x=w^{-1}$ by Proposition 5.1 and induction on $l(x w)$ applied to the fact that $(x w) \cdot \lambda=\lambda$, and we have $\varsigma=w \cdot \nu$ and $\varsigma-w \cdot \lambda=w \cdot \nu-w \cdot \lambda=w(\nu-\lambda)=w \eta$ and $\left(L(\eta) \otimes_{K} M(w \cdot \lambda): M(w \cdot \nu)\right)=\operatorname{dim} L(\eta)_{w \eta}=1$. On the other hand, if we never have equality then $x \cdot \zeta<\nu$ for all $x \in W$, and by Proposition 5.2 we have $\varsigma=w^{\prime} \cdot \chi$ for some $\chi<\nu$ with $\chi+\eta \in P^{+}$.

COROLlaRY 7.3. Let $\mu+\rho \in P^{+}$with $\mu \leq \lambda$, and let $w \in W$. Then

$$
T_{\lambda}^{\nu} M(w \cdot \mu)=0 \quad \text { if } \mu<\lambda,
$$

and

$$
T_{\lambda}^{\nu} M(w \cdot \mu)=M(w \cdot \nu) \quad \text { if } \mu=\lambda .
$$

ProOF. Since $L(\eta) \otimes_{K} M(w \cdot \mu)$ has a VS as described in the proposition, this follows from Proposition 6.1.

COROllary 7.4. Let $\mu+\rho \in P^{+}$with $\mu \leq \lambda$, and let $w \in W$. Then $L(\eta) \otimes_{K}$ $L(w \cdot \mu)$ has a filtration of submodules $0=L_{0} \subset L_{1} \subset \cdots$ with $L(\eta) \otimes_{K} L(w \cdot \mu)=$ $\bigcup_{i \geq 0} L_{i}$ such that each $L_{i} / L_{i-1}(i=1,2, \ldots)$ is either zero or a highest weight module of weight $w_{i} \cdot \chi_{i}$ for some $w_{i} \in W$ and some $\chi_{i} \leq \nu$ with $\chi_{i}+\rho \in P^{+}$. Furthermore, if $\mu<\lambda$ then all $\chi_{i}<\nu$, and if $\mu=\lambda$ then we may arrange to have $\chi_{1}=\nu$ and $w_{1}=w$ and all other $\chi_{i}<\nu$.

ProOF. Recall the sets $S_{2}=W \cdot \nu$ and $S_{3}=\{\varsigma \mid x \cdot \varsigma<\nu$ for all $w \in W\}$ introduced in $\S 5$. By Lemma $5.5, \operatorname{Hom}(M(w \cdot \nu), M(\varsigma))=0$ for all $\varsigma \in S_{3}$. By Proposition $7.2, L(\eta) \otimes_{K} M(w \cdot \mu)$ has a VS $0=M_{0} \subset M_{1} \subset \cdots$, and by the description of the Verma factors given there, together with Proposition 5.1, we see that if $\mu<\lambda$ then all the Verma factors $M(\varsigma)$ satisfy $\varsigma \in S_{3}$, and if $\mu=\lambda$ then all the Verma factors $M(\varsigma)$ except one satisfy $\varsigma \in S_{3}$, and the one exception is $M(w \cdot \nu)$. Thus, in case $\mu=\lambda$ we may arrange to have $M_{1} \cong M(w \cdot \nu)$, by Lemma 4.2 .

Now $L(\eta) \otimes_{K}$ - is an exact functor, so there is an epimorphism

$$
\phi: L(\eta) \otimes_{K} M(w \cdot \mu) \rightarrow L(\eta) \otimes_{K} L(w \cdot \mu) .
$$

Setting $L_{i}=\phi\left(M_{i}\right)$ gives the result.

COROLlaRY 7.5. Let $\mu+\rho \in P^{+}$with $\mu<\lambda$, and let $w \in W$. Then $L(\eta) \otimes_{K}$ $L(w \cdot \mu)$ has no subquotients of the form $L(x \cdot \nu)$ with $x \in W$.

PrOOF. This follows from Proposition 4.1, Lemma 5.5, and the filtration given in Corollary 7.4.

For the next proposition, we need the notion of a contravariant form on a $\mathfrak{g}$ module $M$. This is a symmetric bilinear form $(-,-)$ such that $\left(x \cdot m_{1}, m_{2}\right)=$ $\left(m_{1}, \sigma(x) \cdot m_{2}\right)$ for all $x \in \mathfrak{g}$ and all $m_{1}, m_{2} \in M$, where $\sigma: \mathfrak{g} \rightarrow \mathfrak{g}$ is the involutive antiautomorphism introduced in the definition of $\mathfrak{g}$. It is known (cf., for example, [5]), that if $M$ is a highest weight module, then $M$ has a unique contravariant form 
up to scalar multiples, and the radical of a nonzero contravariant form on $M$ is its unique maximal submodule. In particular, any irreducible highest weight module has a nondegenerate contravariant form.

Another idea we will need for the next proposition is as follows. If $M$ is a weight module over $\mathfrak{g}$, let $M^{\#}$ be the $\mathfrak{g}$-module whose underlying vector space is the subspace of $M^{*}$ (the dual vector space of $M$ ) consisting of those $f: M \rightarrow K$ which vanish on all but finitely many weight spaces of $M$, and whose $\mathfrak{g}$-module structure is defined by $(x \cdot f)(m)=f(\sigma(x) \cdot m)$ for all $x \in \mathfrak{g}, f \in M^{\#}$, and $m \in M$. It is well known that $M^{\#}$ has the same local composition factors as $M$ (in reverse order). This follows easily from the fact that $\operatorname{ch} M^{\#}=\operatorname{ch} M$ for any weight module $M$.

Proposition 7.6. Let $w \in W$. Then $L(\eta) \otimes_{K} L(w \cdot \lambda)$ either has no subquotients of the form $L(x \cdot \nu)$ with $x \in W$, or else it has $L(w \cdot \nu)$ as a subquotient with multiplicity one and no other subquotients of the form $L(x \cdot \nu)$ with $x \in W$.

ProOF. Since $L(\eta)$ and $L(w \cdot \lambda)$ each have nondegenerate contravariant forms, so does $L(\eta) \otimes_{K} L(w \cdot \lambda)$, and we denote this form by $(-,-)$. Let $0=L_{0} \subset L_{1} \subset \cdots$ be the filtration of $L(\eta) \otimes_{K} L(w \cdot \lambda)$ given in Corollary 7.4. Since by Proposition 4.1 and Lemma 5.5 we cannot have $L(x \cdot \nu)$ occurring as a subquotient of any $L_{1} / L_{i-1}$ for $i>1$, we have that $\left(L(\eta) \otimes_{K} L(w \cdot \lambda): L(x \cdot \nu)\right)=\left(L_{1}: L(x \cdot \nu)\right)$ for all $x \in W$. Thus, if $L_{1}=0$ we are done. Assume $L_{1} \neq 0$. Then $\left(L_{1}: L(w \cdot \nu)\right)=1$, since $L_{1}$ has highest weight $w \cdot \nu$. Let $M$ be the maximal submodule of $L_{1}$. We will be done if we can show that $(M: L(x \cdot \nu))=0$ for all $x \in W$. Since $M$ is in the radical of the restriction of $(-,-)$ to $L_{1}$, if $m \in M$ and $\bar{v}=v+L_{1} \in\left(L(\eta) \otimes_{K} L(w \cdot \lambda)\right) / L_{1}$ then $(m, v)$ is independent of the choice of coset representative $v$ for $\bar{v}$. Thus, we may define a linear map $\phi: M \rightarrow\left(\left(L(\eta) \otimes_{K} L(w \cdot \lambda)\right) / L_{1}\right)^{*}$ by $\phi(m)(\bar{v})=(m, v)$. Since the form is contravariant, $\phi$ is actually a $g$-module homomorphism $\phi: M \rightarrow$ $\left(\left(L(\eta) \otimes_{K} L(w \cdot \lambda)\right) / L_{1}\right)^{\#}$. Also, since $(-,-)$ is nondegenerate, $\phi$ is injective, so that if $L(x \cdot \nu)$ is a subquotient of $M$ then it is also a subquotient of $\left(L(\eta) \otimes_{K} L(w \cdot \lambda)\right) / L_{1}$, which cannot happen by Proposition 4.1 and Lemma 5.5. Thus $(M: L(x \cdot \nu))=0$ for all $x \in W$.

This result will be strengthened in the course of the proof of the following theorem.

THEOREM 7.7. Let $\lambda, \eta \in P^{+}$with $\lambda+\eta=\nu$. For any $w_{1}, w_{2} \in W$,

$$
\left(M\left(w_{1} \cdot \nu\right): L\left(w_{2} \cdot \nu\right)\right)=\left(M\left(w_{1} \cdot \lambda\right): L\left(w_{2} \cdot \lambda\right)\right) \text {. }
$$

ProOF. By Proposition 7.2, $L(\eta) \otimes_{K} M\left(w_{1} \cdot \lambda\right)$ satisfies the hypotheses of Theorem 6.9. Thus, from Corollary 7.3 we have

$$
\begin{aligned}
\left(M\left(w_{1} \cdot \nu\right): L\left(w_{2} \cdot \nu\right)\right) & =\left(T_{\lambda}^{\nu} M\left(w_{1} \cdot \lambda\right): L\left(w_{2} \cdot \nu\right)\right) \\
& =\left(W_{0}^{\nu}\left(L(\eta) \otimes_{K} M\left(w_{1} \cdot \lambda\right)\right): L\left(w_{2} \cdot \nu\right)\right) \\
& =\left(L(\eta) \otimes_{K} M\left(w_{1} \cdot \nu\right): L\left(w_{2} \cdot \nu\right)\right) .
\end{aligned}
$$

Let $0=M_{0} \subset M_{1} \subset \cdots \subset M_{n}=M\left(w_{1} \cdot \lambda\right)$ be an LCS for $M\left(w_{1} \cdot \lambda\right)$ at $w_{2} \cdot \nu-\eta$, with factors $F_{i}=M_{i} / M_{i-1}$ for $i=1, \ldots, n$. Observe that $w_{2} \cdot \nu-\eta \leq w_{2} \cdot \nu-w_{2} \eta=$ $w_{2} \cdot(\nu-\eta)=w_{2} \cdot \lambda$, so that this is also an LCS for $M\left(w_{1} \cdot \lambda\right)$ at $w_{2} \cdot \lambda$. Then we have the filtration

$$
\begin{aligned}
0 & =L(\eta) \otimes_{K} M_{0} \subset L(\eta) \otimes_{K} M_{1} \subset \cdots \subset L(\eta) \otimes_{K} M_{n} \\
& =L(\eta) \otimes_{K} M\left(w_{1} \cdot \lambda\right)
\end{aligned}
$$


with factors $L(\eta) \otimes_{K} M_{i} / L(\eta) \otimes_{K} M_{i-1} \cong L(\eta) \otimes_{K} F_{i}$ for $i=1, \ldots, n$. Thus

$$
\left(M\left(w_{1} \cdot \nu\right): L\left(w_{2} \cdot \nu\right)\right)=\sum_{i=1}^{n}\left(L(\eta) \otimes_{K} F_{i}: L\left(w_{2} \cdot \nu\right)\right) .
$$

Now, if $F_{i}$ has no weights $\geq w_{2} \cdot \nu-\eta$, then $L(\eta) \otimes_{K} F_{i}$ has no weights $\geq w_{2} \cdot \nu$, so that $\left(L(\eta) \otimes_{K} F_{i}: L\left(w_{2} \cdot \nu\right)\right)=0$. On the other hand, if $F_{i} \cong L(\chi)$, then by Corollary 7.5 and Proposition 7.6, we have $\left(L(\eta) \otimes_{K} F_{i}: L\left(w_{2} \cdot \nu\right)\right)=0$ unless $\chi=w_{2} \cdot \lambda$, in which case $\left(L(\eta) \otimes_{K} L\left(w_{2} \cdot \lambda\right): L\left(w_{2} \cdot \nu\right)\right)=0$ or 1 , independently of $i$ and independently of $w_{1}$. To summarize,

$$
\begin{aligned}
& \left(M\left(w_{1} \cdot \nu\right): L\left(w_{2} \cdot \nu\right)\right)=\sum_{i=1}^{n}\left(L(\eta) \otimes_{K} F_{i}: L\left(w_{2} \cdot \nu\right)\right) \\
& \quad= \begin{cases}\left(M\left(w_{1} \cdot \lambda\right): L\left(w_{2} \cdot \lambda\right)\right) & \text { if }\left(L(\eta) \otimes_{K} L\left(w_{2} \cdot \lambda\right): L\left(w_{2} \cdot \nu\right)\right)=1, \\
0 & \text { if }\left(L(\eta) \otimes_{K} L\left(w_{2} \cdot \lambda\right): L\left(w_{2} \cdot \nu\right)\right)=0 .\end{cases}
\end{aligned}
$$

But for the special case of $w_{1}=w_{2}$, the multiplicity is known to be nonzero, so we must have $\left(L(\eta) \otimes_{K} L\left(w_{2} \cdot \lambda\right): L\left(w_{2} \cdot \nu\right)\right)=1$, and thus $\left(M\left(w_{1} \cdot \nu\right): L\left(w_{2} \cdot \nu\right)\right)=$ $\left(M\left(w_{1} \cdot \lambda\right): L\left(w_{2} \cdot L\right)\right)$ for all $w_{1}, w_{2} \in W$.

COROLlary 7.8. For any $w_{1}, w_{2} \in W$,

$$
\left(T_{\lambda}^{\nu} L\left(w_{1} \cdot \lambda\right): L\left(w_{2} \cdot \nu\right)\right)= \begin{cases}1 & \text { if } w_{1}=w_{2}, \\ 0 & \text { if } w_{1} \neq w_{2} .\end{cases}
$$

PROOF. From the proof of the theorem, $\left(L(\eta) \otimes_{K} L\left(w_{1} \cdot \lambda\right): L\left(w_{1} \cdot \nu\right)\right)=1$, and by Proposition 7.6, $\left(L(\eta) \otimes_{K} L\left(w_{1} \cdot \lambda\right): L\left(w_{2} \cdot \nu\right)\right)=0$ if $w_{1} \neq w_{2}$. But $L(\eta) \otimes_{K} L\left(w_{1} \cdot \lambda\right)$ satisfies the hypotheses of Theorem 6.9 , by Corollary 7.4. Thus

$$
\begin{aligned}
\left(T_{\lambda}^{\nu} L\left(w_{1} \cdot \lambda\right): L\left(w_{2} \cdot \nu\right)\right) & =\left(W_{0}^{\nu}\left(L(\eta) \otimes_{K} L\left(w_{1} \cdot \lambda\right)\right): L\left(w_{2} \cdot \nu\right)\right) \\
& =\left(L(\eta) \otimes_{K} L\left(w_{1} \cdot \lambda\right): L\left(w_{2} \cdot \nu\right)\right)
\end{aligned}
$$

and the result follows.

COROLlaRY 7.9. For any $\nu \in P^{+}$and any $w_{1}, w_{2} \in W$,

$$
\left(M\left(w_{1} \cdot \nu\right): L\left(w_{2} \cdot \nu\right)\right)=\left(M\left(w_{1} \cdot 0\right): L\left(w_{2} \cdot 0\right)\right) .
$$

PROOF. Observe that taking $\lambda=0 \in P^{+}$and $\eta=\nu-\lambda=\nu \in P^{+}$, we may apply Theorem 7.7 to this special case.

THEOREM 7.10. For any $\lambda, \mu \in P^{+}$and any $w_{1}, w_{2} \in W$,

$$
\left(M\left(w_{1} \cdot \lambda\right): L\left(w_{2} \cdot \lambda\right)\right)=\left(M\left(w_{1} \cdot \mu\right): L\left(w_{2} \cdot \mu\right)\right)
$$

ProOF. Applying Corollary 7.9 twice, we have

$$
\left(M\left(w_{1} \cdot \lambda\right): L\left(w_{2} \cdot \lambda\right)\right)=\left(M\left(w_{1} \cdot 0\right): L\left(w_{2} \cdot 0\right)\right)=\left(M\left(w_{1} \cdot \mu\right): L\left(w_{2} \cdot \mu\right)\right) .
$$

\section{REFERENCES}

1. I. N. Bernstein, I. M. Gelfand, and S. I. Gelfand, Differential operators on the base affine space and $a$ study of $\mathfrak{g}$-modules, Lie Groups and Their Representations, Proc. Summer School on Group Representations, János Bolyai Math. Soc., Wiley, 1975, pp. 35-69.

2. V. V. Deodhar, O. Gabber, and V. G. Kac, Structure of some categories of representations of infinite-dimensional Lie algebras, Adv. in Math. 45 (1982), 92-116. 
3. T. Enright, On the fundamental series of a real semisimple Lie algebra: their irreducibility, resolutions and multiplicity formulae, Ann. of Math. (2) 110 (1979), 1-82.

4. H. Garland and J. Lepowsky, Lie algebra homology and the Macdonald-Kac formulas, Invent. Math. 34 (1976), 37-76.

5. J. C. Jantzen, Moduln mit einem höchsten Gewicht, Lecture Notes in Math., vol. 750, Springer, Berlin, 1979.

6. V. G. Kac, Simple irreducible graded Lie algebras of finite growth, Izv. Akad. Nauk SSSR 32 (1968), 1323-1367; English transl., Math. USSR-Izv. 2 (1968), 1271-1311.

7. R. V. Moody, A new class of Lie algebras, J. Algebra 10 (1968), 211-230.

8. W. Neidhardt, The BGG resolution, character and denominator formulas, and related results for Kac-Moody algebras, Ph.D. thesis, Univ. of Wisconsin, Madison, Wisc., 1985.

9. __ The BGG resolution, character and denominator formulas, and related results for KacMoody algebras, Trans. Amer. Math. Soc. 297 (1987), 487-504.

10. _ A translation principle for Kac-Moody algebras, Proc. Amer. Math. Soc. 100 (1987), 395-400.

11. A. Rocha-Caridi and N. Wallach, Projective modules over graded Lie algebras. I, Math. Z. 180 (1982), 151-177.

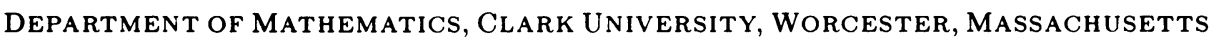
01610

Current address: Department of Mathematics, University of Hawaii - Kapiolani Community College, 4303 Diamond Head Road, Honololu, Hawaii 96816 